\title{
Aspects of the same-sign diboson signature from wino pair production with light higgsinos at the high luminosity LHC
}

\author{
Howard Baer, ${ }^{1, *}$ Vernon Barger, ${ }^{2, \dagger}$ James S. Gainer, ${ }^{3, \ddagger}$ Michael Savoy, ${ }^{1,}$ Dibyashree Sengupta, ${ }^{1, \|}$ and Xerxes Tata ${ }^{3, \uparrow}$ \\ ${ }^{1}$ Department of Physics and Astronomy, University of Oklahoma, Norman, Oklahoma 73019, USA \\ ${ }^{2}$ Department of Physics, University of Wisconsin, Madison, Wisconsin 53706, USA \\ ${ }^{3}$ Department of Physics and Astronomy, University of Hawaii, Honolulu, Hawaii 96822, USA
}

(Received 6 November 2017; published 23 February 2018)

\begin{abstract}
Naturalness arguments applied to simple supersymmetric (SUSY) theories require a set of light higgsinos with mass $\sim|\mu|$ not too far from $m_{h}$. These models have an inverted electroweakino spectrum with $|\mu| \ll M_{2}$ which leads to a rather clean, hadronically quiet, same-sign diboson (SSdB) signature at hadron colliders arising from neutral-plus-charged wino pair production. We improve and expand our earlier studies of this signature for discovering SUSY in natural SUSY models by (i) including backgrounds which were not previously considered and which turn out to be significant, (ii) devising more efficient cuts to successfully contend with these larger backgrounds and determining the discovery reach and exclusion ranges for winos with these cuts, emphasizing projections for the updated integrated luminosity target for HL-LHC of $3 \mathrm{ab}^{-1}$, and (iii) emphasizing the utility of this channel for natural models without gaugino mass unification. We display the kinematic characteristics of the relatively jet-free same sign dilepton $+E_{T}$ events (from leptonic decays of both $\mathrm{Ws}$ ) and find that these are only weakly sensitive to the parent wino mass. We also examine the charge asymmetry in these events and show that its measurement can be used to check the consistency of the wino origin of the signal. Finally, we show that-because the wino branching fractions in natural SUSY are essentially independent of details of the underlying model-a determination of the rate for clean, same-sign dilepton events yields a better than $10 \%$ determination of the wino mass over the entire mass range where experiments at the HL-LHC can discover the wino signal.
\end{abstract}

DOI: 10.1103/PhysRevD.97.035012

\section{INTRODUCTION}

The search for supersymmetry (SUSY) in Run 2 of LHC with $\sqrt{s}=13 \mathrm{TeV}$ and $\sim 36 \mathrm{fb}^{-1}$ of data has resulted in mass limits of $m_{\tilde{g}} \gtrsim 2 \mathrm{TeV}$ [1] and $m_{\tilde{t}_{1}} \gtrsim 0.9 \mathrm{TeV}$ [2]. These rather severe mass limits have led to concern that simple SUSY models may be entering the regime of unnaturalness; if true, such considerations could undermine the entire raison d'etre for weak scale supersymmetry [3]. It should, however, be stressed that conclusions from naturalness regarding upper bounds on sparticle masses $[4,5]$ (limits on stop masses are the most widely discussed) do not apply if the model parameters - often assumed to be independent - turn out to be correlated [6-8].

\footnotetext{
baer@ou.edu

†barger@pheno.wisc.edu

\#jgainer@hawaii.edu

\$savoy@ou.edu

"Dibyashree.Sengupta-1@ou.edu

tata@phys.hawaii.edu
}

Published by the American Physical Society under the terms of the Creative Commons Attribution 4.0 International license. Further distribution of this work must maintain attribution to the author(s) and the published article's title, journal citation, and DOI. Funded by SCOAP.
Quantitative measures of naturalness generally derive from calculations of the fine-tuning of the weak scale, typically represented by the $Z$ boson mass, which is related to other weak-scale SUSY parameters via the Minimal Supersymmetric Standard Model (MSSM) scalar potential minimization condition,

$$
\begin{aligned}
\frac{m_{Z}^{2}}{2}= & \frac{m_{H_{d}}^{2}+\Sigma_{d}^{d}-\left(m_{H_{u}}^{2}+\Sigma_{u}^{u}\right) \tan ^{2} \beta}{\tan ^{2} \beta-1} \\
& -\mu^{2} \sim-m_{H_{u}}^{2}-\mu^{2}-\Sigma_{u}^{u}\left(\tilde{t}_{1,2}\right),
\end{aligned}
$$

where $m_{H_{u, d}}^{2}$ are soft SUSY breaking Higgs mass parameters, $\mu$ is the superpotential Higgs/higgsino mass term, $\tan \beta \equiv$ $v_{u} / v_{d}$ is the ratio of Higgs field vacuum expectation values (vevs), and the $\Sigma_{u}^{u}$ and $\Sigma_{d}^{d}$ terms include a variety of radiative corrections (expressions for these can be found in the Appendix of Ref. [9]). Recently, several of us have suggested using electroweak naturalness as a conservative criterion $[9,10]$ to determine whether a SUSY model spectrum is unnatural. The electroweak naturalness measure is defined as

$\Delta_{\mathrm{EW}}=\max \mid$ each term on the RHS of Eq. $1 \mid /\left(m_{Z}^{2} / 2\right)$. 
Naturalness, then, is the requirement that $\Delta_{\mathrm{EW}}$ is relatively small. Conservatively, requiring $\Delta_{\mathrm{EW}}<30$ implies:

(i) $|\mu| \sim 100-300 \mathrm{GeV}$ (the closer to $m_{Z}$ the better);

(ii) $m_{H_{u}}^{2}$ is radiatively driven from large high scale values to small negative values $\left(\sim-(100-300)^{2} \mathrm{GeV}^{2}\right)$ at the weak scale;

(iii) the magnitude of $\Sigma_{u}^{u}$ is also bounded by about $(300 \mathrm{GeV})^{2}$. This is possible even if stop massesthough bounded above-are in the multi- $\mathrm{TeV}$ range, and gluinos are as heavy as 5-6 TeV [11] (depending on the details of the model). ${ }^{1}$

These conditions are met in a class of "radiatively-driven natural SUSY models" (RNS) [9]. In these SUSY models with low $\Delta_{\mathrm{EW}}$, the largest of the radiative corrections typically come from the top-squark sector contributions to $\Sigma_{u}^{u}$ and are minimized for highly mixed $\mathrm{TeV}$ scale top squarks, a condition which also lifts the Higgs mass, $m_{h}$, into the vicinity of its measured value $\sim 125 \mathrm{GeV}[9,10]$. We emphasize, however, that as Eq. (1) holds in general in the MSSM, the argument that naturalness in the MSSM leads to small $|\mu|$, and concomitantly light higgsinos, ${ }^{2}$ applies whether or not one uses Eq. (2) to define fine-tuning.

We advocate using $\Delta_{\mathrm{EW}}$ for discussions of naturalness. It yields a conservative measure of fine-tuning because it allows for the possibility that model parameters, frequently regarded as independent, might turn out to be correlated once the SUSY breaking mechanism is understood. Ignoring this may lead to an overestimate of the UV sensitivity of $m_{Z}^{2}$ and cause us to prematurely discard perfectly viable models. We also mention that the commonly used Barbieri-Giudice measure $[4,15]$ of fine-tuning reduces to $\Delta_{\mathrm{EW}}$ once appropriate correlations between model parameters are properly implemented [6,7]. That the use of $\Delta_{\mathrm{EW}}$ to assess naturalness is indeed conservative is brought home by explicit examples [7] where the evaluation of $\Delta_{B G}$ with parameter correlations ignored yields $\Delta_{B G}>300 \Delta_{\mathrm{EW}}$.

While naturalness favors a small superpotential $\mu$ parameter, LHC results seem to favor rather heavy gauginos, at least in models with gaugino mass unification (where gaugino masses are related by $M_{1}=M_{2}=M_{3} \equiv m_{1 / 2}$ at the energy scale $Q=m_{\mathrm{GUT}} \simeq 2 \times 10^{16} \mathrm{GeV}$ ). In such models, renormalization group evolution of gaugino masses typically leads to weak scale gaugino masses in the ratio $M_{1}: M_{2}: M_{3} \sim 1: 2: 7$. LHC limits on the gluino mass suggest $M_{3}$ (weak) $\gtrsim 2 \mathrm{TeV}$, which then implies that

\footnotetext{
${ }^{1}$ The limit on the gluino mass arises because radiative corrections from gluino loops raise the stop mass, and as a result $\Sigma_{u}^{u}(\tilde{t})$ becomes too large [12].

${ }^{2}$ Here, we are implicitly assuming that the superpotential parameter, $\mu$, is the dominant source of the higgsino mass. A soft SUSY-breaking contribution to the higgsino mass is possible if there are no additional gauge singlets that couple to higgsinos [13]. In extended frameworks with additional TeV scale fields it is theoretically possible to decouple the higgsino mass from the Higgs boson mass parameter that enters into Eq. (1) [14].
}

the wino mass, $M_{2}, \gtrsim 600 \mathrm{GeV}$, and $M_{1} \gtrsim 300 \mathrm{GeV}$. We should, however, keep in mind that gaugino mass unification is not a prerequisite for naturalness [16], and also that direct limits from electroweak gaugino searches at the LHC should be regarded as independent of those from gluino searches. Indeed searches for wino pair production [17] in simplified models where the charged wino decays via $\tilde{W}^{ \pm} \rightarrow W^{ \pm}+$the lightest supersymmetric particle (LSP), and the neutral wino decays via $\tilde{W}^{0} \rightarrow Z+$ LSP lead to lower bounds $\sim 500 \mathrm{GeV}$ for an LSP mass of about $200 \mathrm{GeV}$. Interestingly, the strongest bound arises from the dilepton-plus-jet channel rather than the clean but rate-suppressed trilepton channel. One might naively expect that as long as the higgsinos are essentially invisible these bounds will continue to apply. However, these bounds weaken considerably in natural SUSY models once the expected branching fractions (see below) for wino decays to light higgsinos are incorporated, and there is essentially no bound if higgsinos are heavier than about $150 \mathrm{GeV}$ but still significantly lighter than the winos. ${ }^{3}$

The inversion of the gaugino-higgsino mass pattern expected in natural supersymmetry has important implications not only for SUSY collider searches but also for dark matter expectations. Since the lightest SUSY particle is expected to be a higgsino-like neutralino, it is thermally underproduced as dark matter. Naturalness in the QCD sector seems to require introduction of an axion [18] which may be expected to constitute the remainder of the dark matter [19]. While the axion and its cousins are well-motivated, we recognize that there are many other possibilities that could lead to the observed dark matter, including out of equilibrium decays of heavy particles into the neutralino LSP.

Though $M_{3}$ is phenomenologically constrained to be $\gtrsim 2 \mathrm{TeV}$, without prejudices from gaugino mass unification the electroweak gaugino mass parameters are relatively unconstrained. If, motivated by naturalness considerations, we assume $|\mu|$ is not hierarchically larger than $M_{Z}$, then it is reasonable to explore LHC prospects for SUSY scenarios with,

$$
|\mu|<M_{1}, \quad M_{2}<M_{3},
$$

where the heavier (wino-like) charginos and neutralinos decay to the light higgsinos via $\tilde{W}_{2}^{ \pm} \rightarrow \tilde{Z}_{1,2}+W^{ \pm}$, $\tilde{W}_{2}^{ \pm} \rightarrow \tilde{W}_{1}^{ \pm}+Z, h$ and $\tilde{Z}_{4} \rightarrow \tilde{Z}_{1,2}+Z, h, \tilde{Z}_{4} \rightarrow \tilde{W}_{1}^{ \pm}+W^{\mp}$.

\footnotetext{
${ }^{3}$ While this is strictly speaking true only for the analysis using chargino-neutralino production alone, in natural SUSY chargino pair production also makes a (subdominant) contribution to the $W Z$ channel. The upper limits on winos of natural SUSY will nonetheless be significantly reduced from those in Ref. [17].

${ }^{4}$ In denoting the winolike neutralino by $\tilde{Z}_{4}$ we have implicitly assumed that the wino is heavier than the bino. This is not really a limitation to the analysis because the binolike state couples rather weakly and so is phenomenologically relatively less important, as long as it is not the LSP.
} 
Although electroweak higgsino pair production processes $p p \rightarrow \tilde{Z}_{i} \tilde{Z}_{j}, \tilde{W}_{1} \tilde{Z}_{i}(i, j=1,2)$ have a large rate for higgsino masses $\sim 150-300 \mathrm{GeV}$, it is difficult to detect these above SM backgrounds unless electroweak gauginos are fortuitously also much lighter than required by naturalness [16]. However, for the generic situation with $\left|M_{1,2}\right| \gg|\mu|$, the higgsino spectra are very compressed, resulting in only relatively soft visible decay products from $\tilde{W}_{1}, \tilde{Z}_{2}$ decays and modest missing transverse energy. One strategy for searching for light higgsinos at the LHC focuses on higgsino pair production in association with a hard jet from initial state QCD radiation which also serves as a trigger. Detailed studies show that although it may be possible to obtain a "signal statistical significance of $5 \sigma$ " above backgrounds after hard cuts, the $S / B$ ratio is just $\sim 1 \%$. It appears to us unlikely that the systematic errors on the QCD background could be reduced to this level [20].

The $S / B$ ratio can be greatly improved by requiring an additional low invariant mass, same flavor, opposite sign soft dilepton pair from $\tilde{Z}_{2} \rightarrow \tilde{Z}_{1} \ell^{+} \ell^{-}$in these hard monojet events. It has been shown that higgsinos up to $200-220 \mathrm{GeV}$ would be detectable at the $5 \sigma$ level at LHC14, assuming an integrated luminosity of $1 \mathrm{ab}^{-1}[21] .^{5}$ Note though that this search will not cover the entire space of SUSY models with $\Delta_{\mathrm{EW}}<30$ even at the high luminosity LHC.

There are several ways to search for superpartners in natural SUSY models. Old favorites like gluino pair production [24] and top-squark pair production [25] remain as important search channels, although now cascade decay events may contain occasional low mass dilepton pairs arising from $\tilde{Z}_{2} \rightarrow \tilde{Z}_{1} \ell^{+} \ell^{-}$decay [26,27]. We have already mentioned the search for soft dileptons in events triggered by a hard monojet (or monophoton). Indeed, the first limits from such a search have been presented by the CMS collaboration in the $m_{\tilde{Z}_{2}}$ vs $m_{\tilde{Z}_{2}}-m_{\tilde{Z}_{1}}$ plane [28].

Yet another distinctive signature for SUSY with light higgsinos (which is the topic of this paper) arises from wino pair production $[26,29]$ via the Feynman diagram shown in Fig. 1: $p p \rightarrow \tilde{W}_{2}^{ \pm} \tilde{Z}_{4}$ followed by $\tilde{W}_{2}^{ \pm} \rightarrow W^{ \pm} \tilde{Z}_{1,2}$ and $\tilde{Z}_{4} \rightarrow$ $W^{ \pm} \tilde{W}_{1}^{\mp}$ decays. Half of the time, the daughter $W$ s will have the same sign, leading to distinctive same sign di-boson (SSdB) plus $E_{T}$ events with no additional jet activity other than from QCD radiation. The subsequent leptonic decays of the $W \mathrm{~s}$ lead to clean same-sign dilepton+ $E_{T}$ events for which the SM backgrounds are very small. We stress that this class of same-sign dilepton events are easily distinguished from those arising from gluino/squark pair production [30] because they are relatively free of accompanying hard jet activity.

\footnotetext{
${ }^{5}$ The detection of pair production of light higgsinos at $e^{+} e^{-}$ colliders with $\sqrt{s}>2 m$ (higgsino) should also be straightforward $[22,23]$, at least for higgsino mass gaps larger than $10 \mathrm{GeV}$.
}

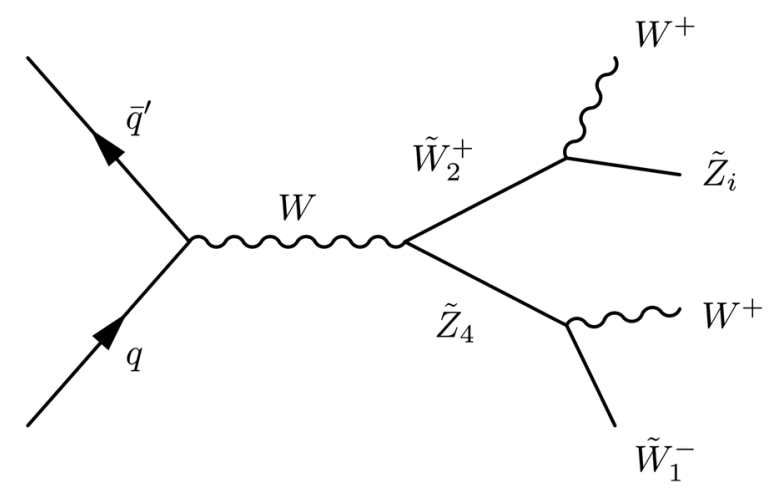

FIG. 1. A Feynman diagram for same-sign diboson production at LHC in SUSY models with light higgsinos.

Some of us have examined this SSdB signature in previous work [26,29]. In these studies, the main SM backgrounds considered were $t \bar{t}, W Z$, and $t \bar{t} W$ production (though $t \bar{t} Z$ and inclusive $W^{ \pm} W^{ \pm}$production from $q q \rightarrow$ $q^{\prime} q^{\prime} W^{ \pm} W^{ \pm}$processes are also mentioned). After a set of cuts to help distinguish the natural SUSY SSdB signal from SM backgrounds, it was found that the background dominantly arose from $t \bar{t} W$ production, and the LHC14 reach was obtained in the two-extra-parameter nonuniversal Higgs (NUHM2) [31] model. ${ }^{6}$ It was emphasized that in models with gaugino mass unification (such as the NUHM2 model), the SUSY reach via the SSdB channel would (for integrated luminosities larger than $\sim 100 \mathrm{fb}^{-1}$ ) exceed the reach via gluino pair production because the winos are only a third as light as gluinos. This assumes that gluinos decay democratically to all generations. In natural SUSY, where gluinos preferentially decay to the third generation, it has been shown that $b$-tagging [32] could be used to further enhance the gluino reach [24] in the $E_{T}$ channel. In Ref. [33], it was emphasized that for natural SUSY models with gaugino mass unification, the $p p \rightarrow$ $\tilde{Z}_{1} \tilde{Z}_{2} j$ reaction followed by $\tilde{Z}_{2} \rightarrow \ell^{+} \ell^{-} \tilde{Z}_{1}$ decay, combined with the SSdB channel, would cover the majority of natural SUSY parameter space with $\Delta_{\mathrm{EW}}<30$ at the high luminosity LHC. This conclusion no longer obtains in string-motivated models such as natural generalized mirage mediation [34] or the minilandscape [35] where the compressed spectrum of gauginos may allow for both wino and gluino masses beyond HL-LHC reach even while maintaining naturalness.

In the current paper, we revisit the SSdB signature from wino pair production in SUSY models with light higgsinos, making a number of important improvements. First, we expand upon earlier calculations by explicitly

\footnotetext{
${ }^{6}$ Since the NUHM2 model allows the soft terms $m_{H_{u}}^{2}$ and $m_{H_{d}}^{2}$ to be traded for weak scale inputs $\mu$ and $m_{A}$, it is easy to generate natural SUSY models by inputting low values of $|\mu| \sim$ 100-300 GeV.
} 
including several additional SM background processes: (1) $W W j j$ production, (2) $t \bar{t} Z$ production, (3) $t \bar{t} t \bar{t}$ production and (4) $W W W$ production. ${ }^{7}$ Second, we focus on the updated integrated luminosity target for the HL-LHC, namely $3000 \mathrm{fb}^{-1}=3 \mathrm{ab}^{-1}$. Third, we emphasize that the SSdB signature from wino pair production offers an independent discovery channel for natural SUSY models, whether gaugino masses are unified or not. For instance, in anomaly-mediated SUSY breaking (AMSB) models, the gaugino masses are expected to occur in the weak scale ratio of $M_{1}: M_{2}: M_{3} \sim 3.3: 1:-7$. For natural AMSB with $|\mu| \ll M_{2}$, it could be that gluino masses are well above LHC reach while wino masses are quite light: $M_{2} \gtrsim 300 \mathrm{GeV}$. In such a case, the SSdB signature might be a robust discovery channel even if gluinos are too heavy to be detected. Since we do not assume gaugino mass unification, we present results in terms of the physical wino mass rather than, e.g., in terms of $m_{1 / 2}$.

In addition to presenting projections for the $5 \sigma$ reaches for the discovery of winos in this channel for various values of the wino mass $m_{\tilde{W}_{2}}$ and the values of $m_{\tilde{W}_{2}}$ that can be expected to be excluded at $95 \%$ confidence level, we also analyze the prospects for wino mass measurement. We point out that using rate information, we can measure the wino mass at better than the $10 \%$ level over its entire discovery range. We show that if there is an excess in the clean SS dilepton sample, a determination of the charge asymmetry would provide an important consistency check. We also examine various kinematic distributions that may reveal characteristic features of the SSdB events. We find that although these distributions in themselves are not strongly sensitive to the wino mass, they may still be useful in a multivariate approach for extracting $M_{2}$.

We discuss our calculation of wino pair production, along with the expected wino decay patterns in natural SUSY and describe our simulation of signal and background processes in Sec. II. The analysis cuts that we suggest for optimizing the SSdB signal at the HL-LHC are described in Sec. III. In Sec. IV we show our projections of the discovery and exclusion reach for winos in the SSdB channel, while various characteristics of signal events are discussed in Sec. V. In Sec. VI, we examine the precision with which the wino mass may be extracted from the SSdB signal rate. Our conclusions are presented in Sec. VII.

\footnotetext{
${ }^{7}$ In addition, our current calculations adopt MADGRAPH [36] and PYTHIA [37] for signal/background calculations and DELPHES [38] for our LHC detector simulation. While it is not obvious that DELPHES/ PYTHIA is an improvement over our previous use of the ISAJET detector simulation, the relative consistency of our new results with our previous results (when direct comparisons can be made) does provide a check on possible systematic errors.
}

\section{EVALUATION OF SIGNAL AND BACKGROUND CROSS SECTIONS}

\section{A. Signal production cross sections}

Since the SSdB signature from pair production of winos is the subject of this study, we begin by showing in Fig. 2 the leading order (LO) and next-to-leading order (NLO) production cross sections for various wino pair production processes-as solid and dashed curves respectively. These cross sections are calculated for the $\sqrt{s}=14 \mathrm{TeV}$ LHC using the PROSPINO computer code [39] and are plotted with respect to the charged wino mass, $m_{\tilde{W}_{2}}$. Since we will also be interested in examining the lepton charge asymmetry, we also show separately the cross sections for $p p \rightarrow$ $\tilde{W}_{2}^{+} \tilde{Z}_{4}$ (red curves) and for $p p \rightarrow \tilde{W}_{2}^{-} \tilde{Z}_{4}$ (green curves).

Note that the $\tilde{W}_{2}^{+} \tilde{Z}_{4}$ cross section typically exceeds the cross section for $\tilde{W}_{2}^{-} \tilde{Z}_{4}$ by a factor $\sim 3-4$. This charge asymmetry in production cross section arises from the preponderance of valence $u$ quarks in the proton versus valence $d$ quarks and increases with $m_{\tilde{W}_{2}}$ due to the growing importance of valence quark over sea quark annihilation as the sampled parton fractional momentum, $x_{F}$, increases. This results in a preponderance of ++ over -- dilepton events as we shall see below.

The charged wino pair production cross section $p p \rightarrow$ $\tilde{W}_{2}^{+} \tilde{W}_{2}^{-}$(blue curves) lies in between the $\tilde{W}_{2}^{+} \tilde{Z}_{4}$ and $\tilde{W}_{2}^{-} \tilde{Z}_{4}$ curves. The black curves denote the cross sections for the summed wino pair production channels, which vary from the tens of fb level for $m_{\tilde{W}_{2}} \sim 600 \mathrm{GeV}$ to $\sim 10^{-2} \mathrm{fb}$ for $m_{\tilde{W}_{2}} \sim 1.6 \mathrm{TeV}$. These wino pair production cross sections hardly vary with respect to $\mu$ (or $\tan \beta$ or $m_{\tilde{q}}$ ) as can be seen from Figs. 4 and 5 of Ref. [26], since the winos couple directly to $W^{ \pm}$via the large $S U(2)_{L}$ coupling, $g$, according to the interactions described in Eq. (8.102) of Ref. [40], and since the higgsino admixture in the winolike state is small.

\section{B. Wino branching fractions}

The $\tilde{W}_{2}$ and $\tilde{Z}_{4}$ branching fractions are calculated using ISAJET 7.85 [41] and have been shown in Refs. [26,29]. We remind the reader that for natural SUSY with light higgsinos, the branching ratios for $\tilde{W}_{2}^{+} \rightarrow \tilde{Z}_{1,2} W^{+}, \tilde{W}_{1}^{+} Z$ and $\tilde{W}_{1}^{+} h$ decays each rapidly asymptote to $\sim 25 \%$ for heavy winos with only small branching fractions to the binolike $\tilde{Z}_{3}$. Likewise, the branching fractions for $\tilde{Z}_{4} \rightarrow \tilde{W}_{1}^{+} W^{-}, \quad \tilde{W}_{1}^{-} W^{+}, \tilde{Z}_{1,2} Z$ and $\tilde{Z}_{1,2} h$ are also each $\sim 25 \%$ for $|\mu| \ll\left|M_{2}\right|$.

These simple decay patterns can be analytically understood in the limit that the $\tilde{W}_{1}$ and $\tilde{Z}_{1,2}$ are mostly higgsinolike, and $\tilde{W}_{2}$ and one of $\tilde{Z}_{3}$ or $\tilde{Z}_{4}$ is mostly a wino (with the other neutralino being dominantly a bino). As already mentioned, the binolike neutralino couples to the wino only via its small higgsino component, so decays to it are 


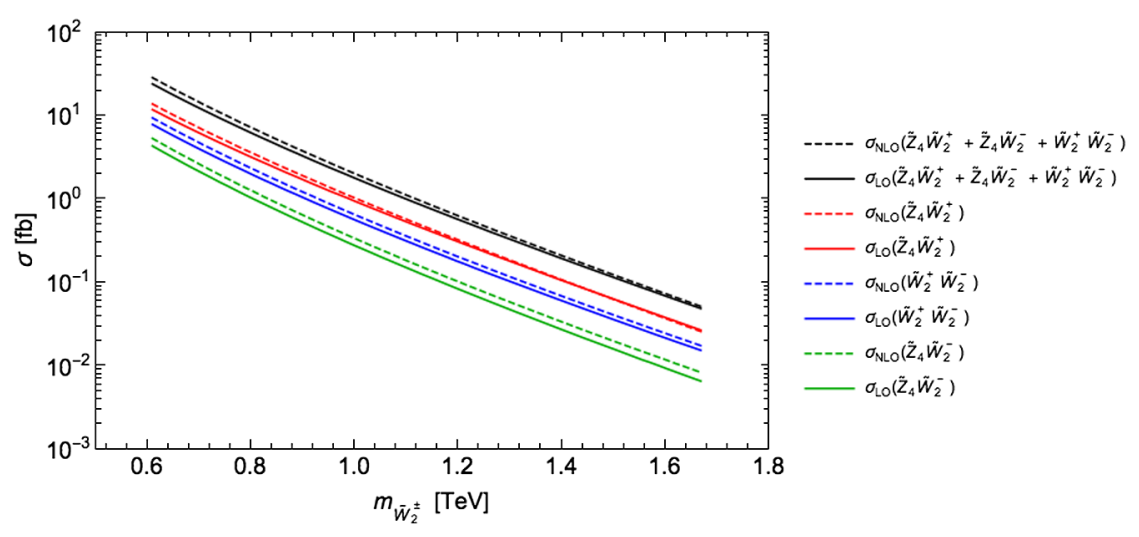

FIG. 2. Leading order (solid) and next-to-leading order (dashed) cross sections for various wino pair production processes at the LHC with $\sqrt{s}=14 \mathrm{TeV}$ versus charged wino mass $m_{\tilde{W}_{2}}$. The neutral wino mass $m_{\tilde{Z}_{4}} \simeq m_{\tilde{W}_{2}}^{ \pm} \sim M_{2}$.

dynamically suppressed even if they are kinematically allowed. In natural SUSY, we are interested in the case $\mu^{2} \ll M_{2}^{2}$, and medium to large $\tan \beta$ values, typically with $\tan \beta>\left|M_{2} / \mu\right|$. In this case, it is straightforward to check that the chargino mixing angle $\gamma_{L} \sim-\gamma_{R} \frac{\mu}{M_{2}}$ (we use the notation of Ref. [40]) so that $\gamma_{L}$ can be ignored compared to $\gamma_{R}$. The small gaugino components of the higgsinolike states and the higgsino components of the winolike states can be evaluated to lowest order in the gaugino-higgsino mixing angles, and the relevant couplings and partial widths for the various decays obtained from the expressions in Appendix B of Ref. [40]. We then find

$$
\begin{aligned}
\Gamma\left(\tilde{W}_{2} \rightarrow \tilde{Z}_{1} W\right) & \simeq \Gamma\left(\tilde{W}_{2} \rightarrow \tilde{Z}_{2} W\right) \\
& \simeq \Gamma\left(\tilde{W}_{2} \rightarrow \tilde{W}_{1} Z\right) \simeq \Gamma\left(\tilde{W}_{2} \rightarrow \tilde{W}_{1} h\right) \\
& \simeq \frac{g^{2}}{64 \pi} m_{\tilde{W}_{2}}, \\
\Gamma\left(\tilde{Z}_{4} \rightarrow \tilde{W}_{1}^{-} W^{+}\right) & \simeq \Gamma\left(\tilde{Z}_{4} \rightarrow \tilde{W}_{1}^{+} W^{-}\right) \simeq \Gamma\left(\tilde{Z}_{4} \rightarrow \tilde{Z}_{1,2} Z\right) \\
& \simeq \Gamma\left(\tilde{Z}_{4} \rightarrow \tilde{Z}_{1,2} h\right) \simeq \frac{g^{2}}{64 \pi} m_{\tilde{Z}_{4}},
\end{aligned}
$$

where, to illustrate our point, we have retained only the largest mass terms in the expressions for the partial widths. This is a good approximation when higgsinos are much lighter than the winos. In our numerical calculation, we retain the full expressions, of course. In the last of these equations we have assumed that $\tilde{Z}_{4}$ is the wino-like state. Also, the neutral wino decay widths to $Z$ or $h$ are the summed widths to both higgsino-like states. ${ }^{8}$ If other decay

\footnotetext{
${ }^{8}$ The reader may wonder why the decay rates to Higgs bosons which go via the unsuppressed wino-higgsino-Higgs boson coupling are comparable to the decay rates to vector bosons which can only occur via small mixing angles. The reason is that this suppression is compensated by the enhancement of the amplitude for decays to longitudinal $W$ or $Z$ bosons by a factor $m_{\tilde{W}_{2}, \tilde{Z}_{4}} / M_{W, Z}$, an example of the Goldstone boson equivalence theorem.
}

modes of the wino (e.g., to the bino, to sfermions, or to the heavy Higgs bosons) are kinematically or dynamically suppressed, we obtain the approximately equal branching fractions of $25 \%$ mentioned above. We have checked by a numerical scan that when $|\mu|=150-300 \mathrm{GeV}$, as favored by naturalness, the branching ratios for these modes are well within the 0.23-0.27 range if the wino is heavier than $500 \mathrm{GeV}$ and the bino is not quasi-degenerate with the wino.

Combining decay channels, we find that typically $\sim 1 / 8$ of $\tilde{W}_{2}^{ \pm} \tilde{Z}_{4}$ production events lead to final states with samesign dibosons $W^{+} W^{+}$or $W^{-} W^{-}$. To identify SSdB events, we require leptonic decays of the final state $W$ 's to $e$ or $\mu$ which reduces our overall branching fraction to $\sim 6 \times 10^{-3}$. Thus, although the wino pair production cross sections may be as large as $10 \mathrm{fb}$, the combined signal channel branching fractions lead to relatively small signal rates. Therefore, the SSdB signal channel really becomes the signal of choice only for the very high integrated luminosities projected to be accumulated at the high-luminosity LHC.

\section{Signal benchmark model line}

To make specific predictions for the expected SSdB signal rate, we will adopt a natural SUSY model line using the twoextra-parameter nonuniversal Higgs model NUHM2 [31]. This model allows for direct input of a low $\mu$ parameter as required by naturalness. The model line we adopt is adapted from Ref. [26] and has $m_{0}=5 \mathrm{TeV}, A_{0}=-8 \mathrm{TeV}$, $\tan \beta=10, m_{A}=1.5 \mathrm{TeV}$, and $\mu=150 \mathrm{GeV}$. We will allow the unified gaugino mass parameter $m_{1 / 2}$ to vary from 700 to $1375 \mathrm{GeV}$ which corresponds to $m_{\tilde{g}} \sim$ $1.8-3.2 \mathrm{TeV}$ or $m_{\tilde{W}_{2}} \sim 610-1200 \mathrm{GeV}$. The value of $m_{h}$ is $\sim 125 \mathrm{GeV}$ along the entire model line, while $\Delta_{\mathrm{EW}}$ is $\sim 10-30$, corresponding to $10 \%-3 \%$ EW fine-tuning. Although the NUHM2 model assumes a unification of gaugino mass parameters, this is unimportant for the analysis of the wino signal that we are focussing upon, in the sense that essentially identical results would be obtained in any model with the same value of the wino mass $M_{2}$. While there 
TABLE I. Input parameters and masses in $\mathrm{GeV}$ units for an NUHM2 model SUSY benchmark point labeled Point B with $m_{t}=173.2 \mathrm{GeV}$ and $m_{1 / 2}=800 \mathrm{GeV}$.

\begin{tabular}{lc}
\hline \hline Parameter & Point B \\
\hline$m_{0}$ & 5000 \\
$m_{1 / 2}$ & 800 \\
$A_{0}$ & -8000 \\
$\tan \beta$ & 10 \\
$\mu$ & 150 \\
$m_{A}$ & 1500 \\
$m_{\tilde{g}}$ & 2007.4 \\
$m_{\tilde{u}_{L}}$ & 5170.2 \\
$m_{\tilde{u}_{R}}$ & 5318.4 \\
$m_{\tilde{e}_{R}}$ & 4815.2 \\
$m_{\tilde{t}_{1}}$ & 1470.3 \\
$m_{\tilde{t}_{2}}$ & 3651.2 \\
$m_{\tilde{b}_{1}}$ & 3682.7 \\
$m_{\tilde{b}_{2}}$ & 5051.2 \\
$m_{\tilde{\tau}_{1}}$ & 4740.2 \\
$m_{\tilde{\tau}_{2}}$ & 5075.6 \\
$m_{\tilde{\tau}_{\tau}}$ & 5082.8 \\
$m_{\tilde{W}_{2}}$ & 692.2 \\
$m_{\tilde{W}_{1}}$ & 155.2 \\
$m_{\tilde{Z}_{4}}$ & 703.1 \\
$m_{\tilde{Z}_{3}}$ & 363.1 \\
$m_{\tilde{Z}_{2}}$ & 158.2 \\
$m_{\tilde{Z}_{1}}$ & 142.4 \\
$m_{h}$ & 124.4 \\
$\Omega_{\tilde{Z}_{1}}^{s t} h^{2}$ & 0.008 \\
$\mathrm{BF}(b \rightarrow s \gamma) \times 10^{4}$ & 3.1 \\
$\mathrm{BF}\left(B \mu_{s} \rightarrow \mu^{+}\right) \times 10^{9}$ & 3.8 \\
$\sigma^{S I}\left(\tilde{Z}_{1}, p\right)(\mathrm{pb})$ & $1.5 \times 10^{-4}$ \\
$\sigma^{S D}\left(\tilde{Z}_{1} p\right)(\mathrm{pb})$ & $2.9 \times 10^{-25}$ \\
$\left.\langle\sigma v\rangle\right|_{v \rightarrow 0}\left(\mathrm{~cm}{ }^{3} / \mathrm{sec}\right)$ & 9.3 \\
$\Delta_{\mathrm{EW}}$ & \\
\hline \hline & \\
\hline
\end{tabular}

may be some sensitivity to the bino mass parameter, we remind the reader that the binolike state couples to the winovector boson system only via its small higgsino components, so any decays into this state typically have small branching fractions.

In Table I, we show a listing of various sparticle masses and observables associated with our model line for the benchmark model with $m_{1 / 2}=800 \mathrm{GeV}$, labeled as Point B. ${ }^{9}$ Within the NUHM2 framework, the model point with the $692 \mathrm{GeV}$ wino state $\tilde{W}_{2}$ has $m_{\tilde{g}} \approx 2000 \mathrm{GeV}$ and so is just beyond the current gluino mass limit (from $13 \mathrm{TeV}$ LHC running with $\sim 35 \mathrm{fb}^{-1}$ ). Though the details of most of the SUSY spectrum are unimportant for our present purposes, we note that our sample case (indeed the entire

\footnotetext{
${ }^{9}$ We refer to this as Point $\mathrm{B}$ because we consider three signal benchmark points, labeled $\mathrm{A}, \mathrm{B}$, and $\mathrm{C}$, in order of increasing wino mass.
}

model line) has very heavy first/second generation sfermions, with stops and gluinos in between these and the EW gauginos, while higgsinos are very light. This qualitative pattern is a generic feature of natural SUSY models. We emphasize that while our benchmark model line is in a model with gauge coupling unification, this will have very little (if any) effect on any conclusions we draw about the prospects for discovery, exclusion, or mass measurement of the parent wino. In other words, for the purposes of analysis of the wino signal alone, we can disregard the LHC gluino limit and model cases with lighter winos that may arise in natural models without gaugino mass unification using $m_{1 / 2}$ as a surrogate for the wino mass, $M_{2}$.

\section{SM background cross sections}

In order to assess prospects for observability of the signal, we must have a good understanding of various SM backgrounds that could also lead to the clean same sign dilepton plus $E_{T}$ signature. We have considered backgrounds from $t \bar{t}$, $W Z, t \bar{t} W, t \bar{t} Z, t \bar{t} t \bar{t}, W W W$, and $W^{ \pm} W^{ \pm} j j$ production processes in the SM. Top pair production yields (noninstrumental) backgrounds only if a secondary lepton from top decay is accidently isolated. We use LO event generation from MADGRAPH in our simulation of both signals and backgrounds, but rescale the $\mathrm{LO}$ total cross sections to be in accordance with NLO values found in the literature.

Specifically, we use $953.6 \mathrm{pb}$ as the total NLO cross section for $t \bar{t}$, following Ref. [42]. Reference [43] gives us a $\mathrm{K}$ factor of 1.27 for four-top production. We use 1.88 as the $\mathrm{K}$ factor for associated $W Z$ production following Ref. [44] and 1.24 for the $\mathrm{K}$ factor for $t \bar{t} W$ production following Ref. [45]. ${ }^{10}$ We obtain the $\mathrm{K}$ factor 1.39 for $t \bar{t} Z$ from Ref. [46]; Ref. [47] gives us a K factor of 1.04 for $W W j j .{ }^{11}$ Finally, for the $W W W$ process we use the cross sections in Ref. [48]. In our analyses we use a common $\mathrm{K}$ factor of 2.45 for both $W W W$ processes, which is not appreciably different than the $W^{+} W^{+} W^{-} \mathrm{K}$ factor of 2.38 or the $W^{+} W^{-} W^{-} \mathrm{K}$ factor of 2.59 . We note that these are $\mathrm{K}$ factors for inclusive $W W W$ production; if one imposes a jet veto the $\mathrm{K}$ factor is significantly reduced (to 1.29 for the combined $W W W \mathrm{~K}$ factor). While we do impose a jet multiplicity cut of $n_{\text {jet }} \leq 1$, we choose to be conservative and use the larger value for the $\mathrm{K}$ factor in our calculation of the background.

\footnotetext{
${ }^{10}$ While in Ref. [44], $\mathrm{K}$ factors differ slightly for $W^{+} Z$ and $W^{-} Z$, and in Ref. [45] the $\mathrm{K}$ factors differ slightly for $t \bar{t} W^{+}$and $t \bar{t} W^{-}$, these are very close $\left(1.86\right.$ and 1.92 respectively for $W^{+} Z$ and $W^{-} Z$ and 1.22 and 1.27 for $t \bar{t} W^{+}$and $t \bar{t} W^{-}$respectively), especially when compared with likely theory errors, so we use 1.88 (1.24) as the $\mathrm{K}$ factor for both $W Z(t \bar{t} W)$ processes.

${ }^{11}$ This is the value in Ref. [47] for the two-jet inclusive cross section with factorization and renormalization scales set to $150 \mathrm{GeV}$. If we were to further restrict to one-jet and zero-jet bins (see our analysis cuts, below), the $\mathrm{K}$ factor would move closer to 1 ; we have chosen the larger $\mathrm{K}$ factor to be conservative.
} 
TABLE II. Component background and signal cross sections in $a b$ before any cuts, after $\mathbf{C} \mathbf{1}$ cuts, after $\mathbf{C 1}$ cuts plus a jet veto, and after C2 at LHC14. Also shown is the $K$-factor that we use.

\begin{tabular}{|c|c|c|c|c|c|}
\hline Process & $K$-factor & $\sigma(\mathrm{NLO})(\mathrm{ab})$ & $\mathrm{C} 1$ & $\mathbf{C} \mathbf{1}+n_{\mathrm{jet}} \leq 1$ & $\mathrm{C} 2$ \\
\hline SUSY (Point B) & 1.25 & $1.55 \times 10^{4}$ & 28.8 & 20.5 & 16.1 \\
\hline$\overline{t \bar{t}}$ & 1.72 & $9.5 \times 10^{8}$ & 0 & 0 & 0 \\
\hline$W Z$ & 1.88 & $5.2 \times 10^{7}$ & 0 & 0 & 0 \\
\hline$t \bar{t} W$ & 1.24 & $5.2 \times 10^{5}$ & 11.1 & 4.7 & 1.7 \\
\hline$t \bar{t} Z$ & 1.39 & $8.8 \times 10^{5}$ & 7.9 & 0.9 & 0 \\
\hline$t \bar{t} t \bar{t}$ & 1.27 & $1.1 \times 10^{4}$ & 0.6 & 0. & 0. \\
\hline$W W W$ & 2.45 & $3.2 \times 10^{5}$ & 7.4 & 5.6 & 2.3 \\
\hline$W W j j$ & 1.04 & $3.9 \times 10^{5}$ & 7.0 & 0.8 & 0.8 \\
\hline Total BG & $\cdots$ & $1.0065 \times 10^{9}$ & 34.1 & 11.9 & 4.8 \\
\hline
\end{tabular}

These $\mathrm{K}$ factors and NLO cross sections for the underlying fundamental SM processes are shown in columns 2 and 3 of Table II, together with the corresponding information for the signal benchmark Point B. These are, of course, the raw production cross sections for the various final states; various branching fractions and detection efficiencies have to be folded in to obtain the signal and background cross sections. We see that even the various $2 \rightarrow 3$ and $2 \rightarrow 4$ SM processes have potentially larger rates than the signal, so we may anticipate that we will require relatively stringent selection cuts to make the signal observable.

\section{E. Event simulation}

To simulate SSdB signal events, we first generate the SUSY spectrum as a Les Houches Accord (LHA) file using ISAJET 7.85 [41]. We then feed the LHA information to MAdGRAPH/MadEvent 2.3.3 [36] which is interfaced with PYTHIA 6.4 [37] for parton showering and hadronization. The generated events are passed to DeLPHES 3.3.0 [38] for fast detector simulation, where we utilize the default "CMS" parameter card for version 3.3.0 with the modifications listed below.

(1) We require jets to have transverse energy $E_{T}$ (jet) $>$ $50 \mathrm{GeV}$ and pseudorapidity $\mid \eta($ jet $) \mid<3.0$.

(2) The electromagnetic calorimeter (ECAL) energy resolution is set to $3 \% / \sqrt{E} \oplus 0.5 \%$, while the hadronic calorimeter (HCAL) energy resolution is taken to be $80 \% / \sqrt{E} \oplus 3 \%$ for $|\eta|<2.6$ and $100 \% / \sqrt{E} \oplus 5 \%$ for $|\eta|>2.6$, where $\oplus$ denotes combination in quadrature.

(3) The jet energy scale correction is turned off.

(4) The anti- $k_{T}$ jet algorithm [49] is utilized, but using $R=0.4$ rather than the default $R=0.5$. (Jet finding in Delphes is implemented via FastJet [50].) One motivation for choosing $R=0.4$ in the jet algorithm is to facilitate comparison with CMS $b$-tagging efficiencies [51].

(5) We performed jet flavor association using our own module which implements the "ghost hadron" procedure [52] which allows the assignment of decayed hadrons to jets in an unambiguous manner. We use this module to aid in $b$-tagging, specifically in determining whether jets contain $B$ hadrons. When a jet contains a $B$ hadron in which the $b$ quark will decay at the next step of the decay, then if this $B$ hadron lies within $|\eta|<3.0$ and $E_{T}>15 \mathrm{GeV}$, we identify this $b$-jet as a "truth $b$-jet." We $b$-tag truth $b$-jets with $|\eta|<1.5$ with an efficiency of $60 \%$. We also $b$-tag jets which are not truth $b$-jets with $|\eta|<1.5$ with an efficiency of $1 / X$ where $X=150$ for $E_{T}<100 \mathrm{GeV}, X=50$ for $E_{T}>250 \mathrm{GeV}$ and $X$ is found from a linear interpolation for $100 \mathrm{GeV}<E_{T}<250 \mathrm{GeV}^{12}$ We have checked [24] that our $b$-jet tagging algorithm yields good agreement with the $b$-tagging efficiencies and mistag rates in Ref. [51]; specifically it gives results intermediate between the CMS "medium" and "tight" $b$-tagging algorithms.

(6) "Tau tagging", i.e., identifying objects as taus, is not used.

(7) The lepton isolation modules were modified to allow us to adopt the isolation criterion that the sum of $E_{T}$ of physics objects in a cone with $\Delta R<0.2$ about the lepton direction is less than $\min \left(5 \mathrm{GeV}, 0.15 E_{T}(\ell)\right)$, where $E_{T}(\ell)$ is the transverse energy of the lepton. (DELPHES 3.3.0 did not allow the minimum of these two thresholds to be used rather than using either a fixed value of $E_{T}$ or a fraction of the lepton $E_{T}$.)

\section{ANALYSIS CUTS TO ENHANCE SUSY SSDB SIGNAL}

\section{A. Initial selection cuts (C1)}

We begin by imposing the selection cuts, listed below, that were suggested in Refs. [26,29] to enhance same sign

\footnotetext{
${ }^{12}$ The parameters for this $b$-tagging procedure are based on ATLAS studies of $b$-tagging efficiencies and rejection factors in $t \bar{t} H$ and $W H$ production processes [53].
} 


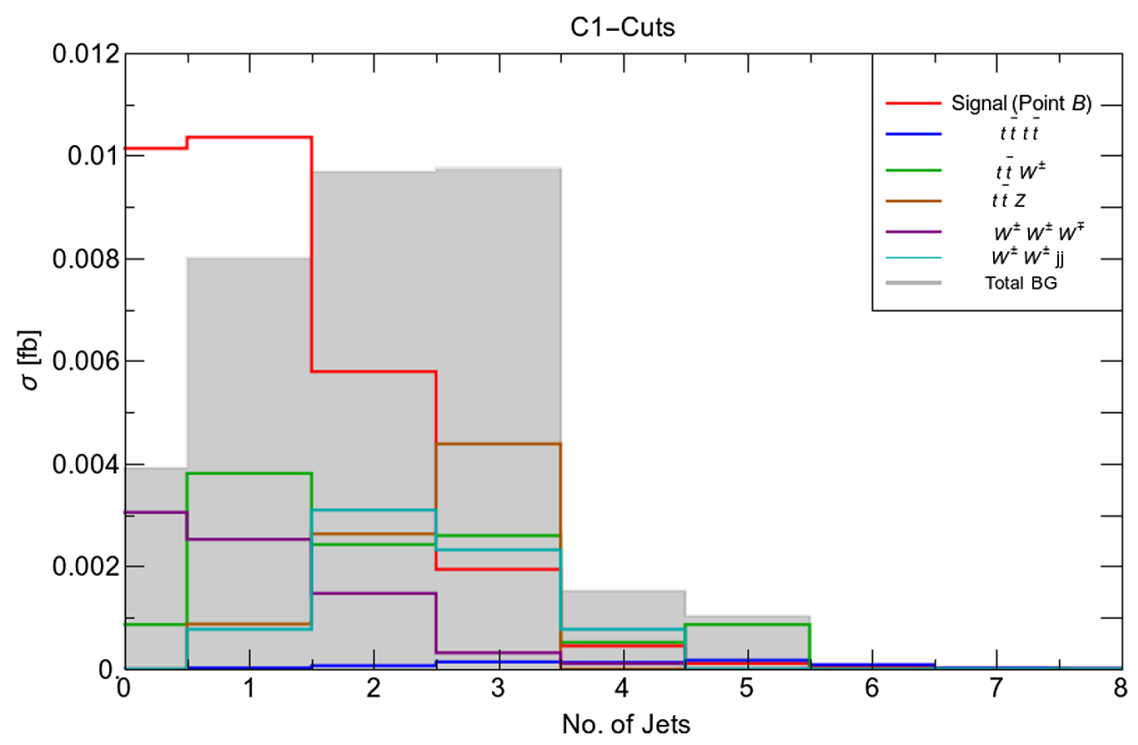

FIG. 3. Distribution of jet multiplicity, $n(j)$, for SSdB events from the Point B signal benchmark point and various SM backgrounds after $\mathbf{C} 1$ cuts.

dilepton events originating in wino production over those coming from SM processes.

(i) Exactly two isolated same-sign leptons with $p_{T}\left(\ell_{1}\right)>20 \mathrm{GeV}$ and $p_{T}\left(\ell_{2}\right)>10 \mathrm{GeV}$. $\left(\ell_{1}\right.$ denotes the higher $p_{T}$ lepton, while $\ell_{2}$ is the lower $p_{T}$ lepton.)

(ii) $n$ (b-jets) $=0$

(iii) $E_{T}>200 \mathrm{GeV}$, and

(iv) $m_{T}^{\min }>175 \mathrm{GeV}$,

where $m_{T}^{\min }=\min \left[m_{T}\left(\ell_{1}, E_{T}, m_{T}\left(\ell_{2}, E_{T}\right)\right]\right.$. We denote these initial cuts as cut set $\mathbf{C} 1$.

The cross sections after these cuts-after folding in various branching fractions and detection efficiencies-for the Point B signal benchmark point and from various SM processes (in ab) are listed in column 4 of Table II. The combined same-sign dilepton cut, large $E_{T}$ cut, and $b$-jet veto serve to severely reduce the $t \bar{t}$ background. Indeed, after these cuts, the analysis of Refs. [26,29] found the dominant background to come from $t \bar{t}$ and $W Z$ production. Any $t \bar{t}$ background events which survive these cuts will likely have one lepton arising from real $W \rightarrow \ell \nu$ decay with the other lepton arising from a semileptonic $b$ decay, which will hence be soft. In such a case, at least to the extent that the $E_{T}$ dominantly arises from the leptonic decay of a single $W$, the transverse mass, $m_{T}\left(\ell, \nu_{\ell}\right)$, is mostly bounded by $m_{W}$ (up to small contamination from off-shell $W \mathrm{~s}, E_{T}$ smearing, and any additional $E_{T}$ from leptonic decays of the $B$-hadron). Thus, the further requirement of $m_{T}^{\min } \gg m_{W}$ should serve to greatly reduce the $t \bar{t}$ and also $W Z$ backgrounds. Here, in accord with Refs. [26,29], we require $m_{T}^{\min }>175 \mathrm{GeV}$; after imposing this cut we are indeed left with no $t \bar{t}$ or $W Z$ backgrounds in our samples. Among the largest backgrounds is $t \bar{t} W$ production, which we find to be a factor of two larger than in Ref. [26]. Unlike the earlier studies, we also find sizable contributions from $t \bar{t} Z$ production as well as from $W W W$ production and $W^{ \pm} W^{ \pm} j j$ production. Summing these sources, we find a total background cross section after C1 cuts of $34 \mathrm{ab}$ in contrast to just $6 \mathrm{ab}$ after the same cuts in Ref. [26]. The cross section for the signal at the benchmark Point B is $29 \mathrm{ab}$, or a little under $5 \sigma$ statistical significance for an integrated luminosity of $1 \mathrm{ab}^{-1}$, and over $8.5 \sigma$ significance with $3 \mathrm{ab}^{-1}$.

\section{B. Optimizing the reach of HL-LHC: Selection cuts C2}

The cut set $\mathbf{C 1}$ was suggested in Refs. [26,29] to determine the reach of LHC14 in the SSdB channel for $100-1000 \mathrm{fb}^{-1}$. Since one of our goals is to project the maximum reach of the HL-LHC for SUSY in the SSdB channel, we attempt to further optimize our cuts.

We begin by noting that the various background processes in Table II with significant cross sections after C1 cuts are all expected to contain additional hard jets, while jet activity in the signal process arises only from initial state QCD radiation (and very soft jets from decay of the heavier higgsinos). We thus anticipate that jet multiplicity will be a useful discriminating variable. ${ }^{13}$ With this motivation we show the expected jet multiplicity, $n(j)$, from signal and background events after the $\mathbf{C 1}$ cuts in Fig. 3. From the solid (red) signal histogram, we see that signal events indeed mainly have $n(j)=0$ or 1 . In contrast, background events, the sum of which is shown by the shaded histogram,

\footnotetext{
${ }^{13}$ In this vein, the scalar sum of jet $E_{T}$ or the ratio of this to the scalar sum of leptonic $E_{T}$ may prove to be even more robust and equally discriminating variables.
} 


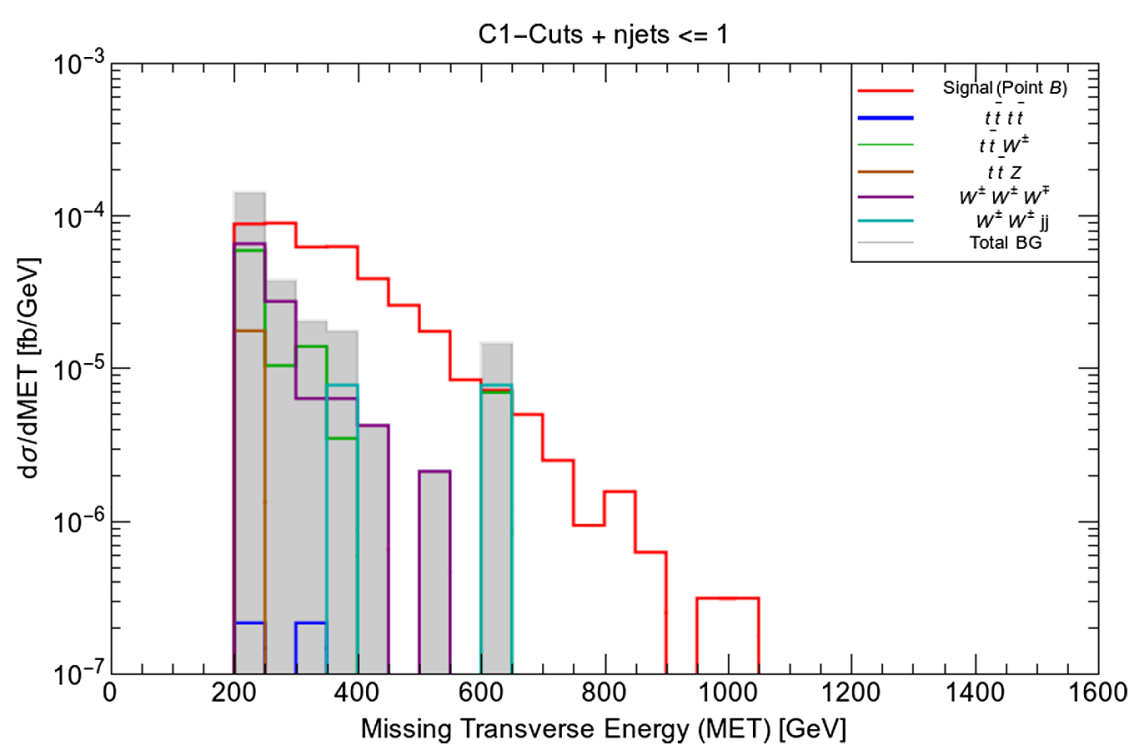

FIG. 4. Distribution of $\mathscr{E}_{T}$ for the signal benchmark Point B and various SM backgrounds in SSdB production after C1 cuts plus the $n(j) \leq 1$ cut.
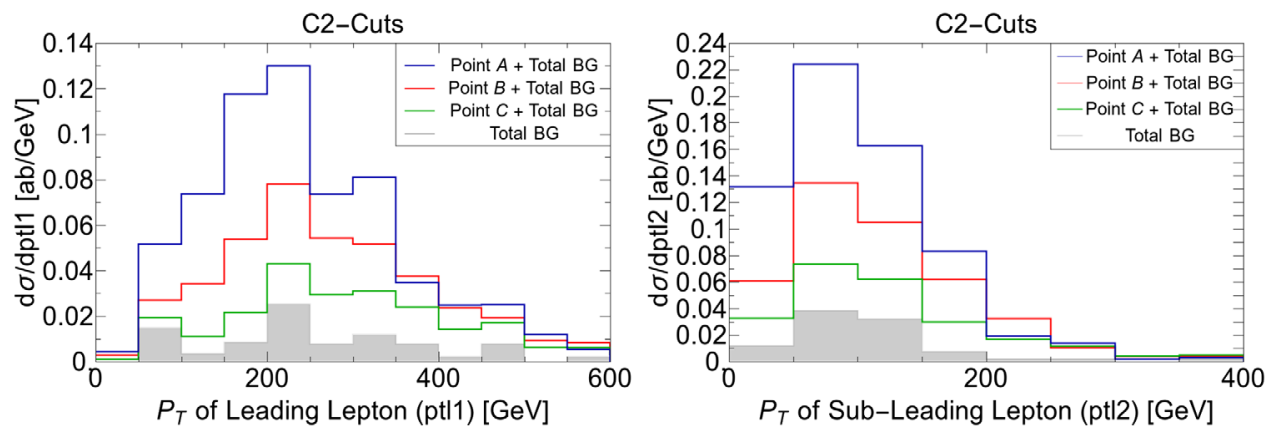

FIG. 5. Distribution of $p_{T}\left(\ell_{1}\right)$ (left frame) and $p_{T}\left(\ell_{2}\right)$ (right frame) for the Point $\mathrm{A}$, Point $\mathrm{B}$, and Point $\mathrm{C}$ benchmarks, which are points along our NUHM2 model line with $m_{\tilde{W}_{2}}=530,692$ and $886 \mathrm{GeV}$, respectively, together with the total SM background after $\mathbf{C} 2$ cuts.

generally have $n(j) \geq 2$. Thus, we apply the additional cut, $n(j) \leq 1$.

The cross sections after cut set $\mathbf{C} \mathbf{1}$ and $n(j) \leq 1$ are listed in column 5 of Table II. ${ }^{14}$ We see that the main background contributions now come from $t \bar{t} W$ and $W W W$ production processes. To further reduce these, we examined several other kinematic distributions including $E_{T}, m_{T}\left(\ell_{1} \ell_{2}, E_{T}^{\prime}\right)$ (the dilepton-plus- $E_{T}$ cluster transverse mass) [54], $m_{T}^{\min }$ and $m_{T 2}$ [55]. The most useful of these turned out to be the $E_{T}$ distribution shown in Fig. 4. From this figure, we see that in the $E_{T}=200-250 \mathrm{GeV}$ bin, the summed background exceeds the signal for Point B, while in higher $E_{T}^{t}$ bins, signal clearly emerges above background. However, care must be taken since our signal rate is already rather

\footnotetext{
${ }^{14}$ The $t \bar{t} W, t \bar{t} Z$ and $W W W$ cross sections have been normalized to their NLO values. Since jet production from these backgrounds occurs already at LO, and initial state shower radiation is already included in our event generation, we expect additional NLO QCD corrections to these backgrounds to be unimportant.
}

small. We elect to make one final cut $E_{T}>250 \mathrm{GeV}$, and label this set of cuts (C1 cuts plus $n(j) \leq 1$, plus $\left.E_{T}>250 \mathrm{GeV}\right)$ as the cut set $\mathbf{C 2}$.

We show the expected $p_{T}$ distributions of the leptons after the $\mathbf{C} 2$ cuts in Fig. 5 for three signal benchmark points along the model line, as well as for the summed SM background. The points have $m_{\tilde{W}_{2}}=530 \mathrm{GeV}$ (Point A), $692 \mathrm{GeV}$ (Point B, already introduced above), and $886 \mathrm{GeV}$ (Point C). We see that the distributions are qualitatively similar, and while the $S / B$ ratio may be slightly improved by requiring harder cuts on the leptons, this would only be at the cost of reducing an already ratelimited signal. We choose, therefore, not to impose any further cuts.

The total background after these cuts is shown in the last column of Table II. We see that almost half this background comes from SM $W W W$ production. We remind the reader of our discussion in Sec. II D, where we mentioned that we have used $K_{W W W}=2.45$, i.e., the value obtained for inclusive $W W W$ production, instead of the much smaller 
value $K_{W W W}=1.29$ one obtains for $W W W$ production with a jet veto. It is very possible that we may have overestimated this background, but we choose to err on the conservative side in our assessment of the discovery prospects of the HL-LHC, the subject of the next section.

\section{DISCOVERY PROSPECTS AT THE HL-LHC}

In Fig. 6, we show the total same sign dilepton signal rate after our final analysis cuts, C2, as a function of the wino mass, $m_{\tilde{W}_{2}}$, (solid blue curve) along with the total SM background (denoted by the dotted red line). We also compute the reach for $5 \sigma$ discovery and $95 \%$ Confidence Level (CL) exclusion for the HL-LHC (using Poisson statistics) with a data sample of $3 \mathrm{ab}^{-1}$. We find that the $5 \sigma$ discovery reach extends to $m_{\tilde{W}_{2}} \sim 860 \mathrm{GeV}$, while the 95\% CL exclusion reach extends to $m_{\tilde{W}_{2}} \sim 1080 \mathrm{GeV}$. As stressed previously, although the model line we have used includes the assumption of gaugino mass unification, our projected reach does not depend on this assumption, but only on $M_{2} \gg|\mu|$, as expected in natural SUSY. In models with gaugino mass unification, the $5 \sigma(95 \% \mathrm{CL})$ reach in $m_{\tilde{W}_{2}}$ correspond to a reach (exclusion) in terms of the

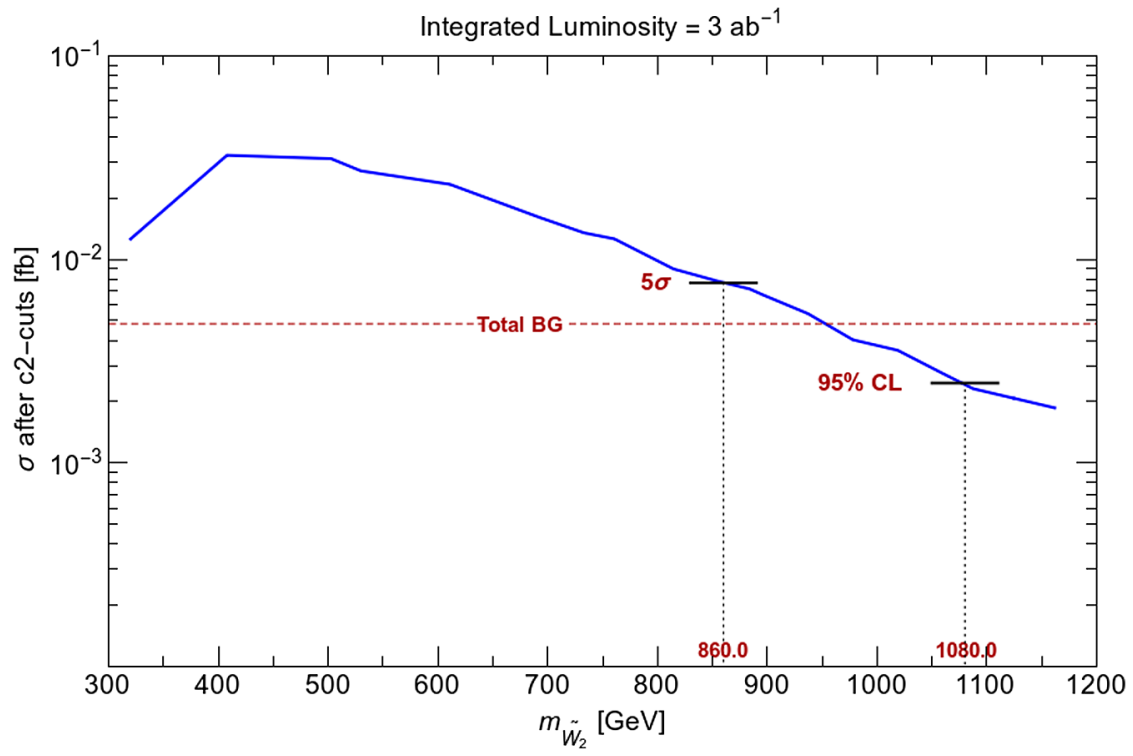

FIG. 6. Cross section for SSdB production after $\mathbf{C} 2$ cuts versus $m$ (wino) at the LHC with $\sqrt{s}=14 \mathrm{TeV}$. We show the $5 \sigma$ and $95 \% \mathrm{CL}$ reach assuming a HL-LHC integrated luminosity of $3 \mathrm{ab}^{-1}$.

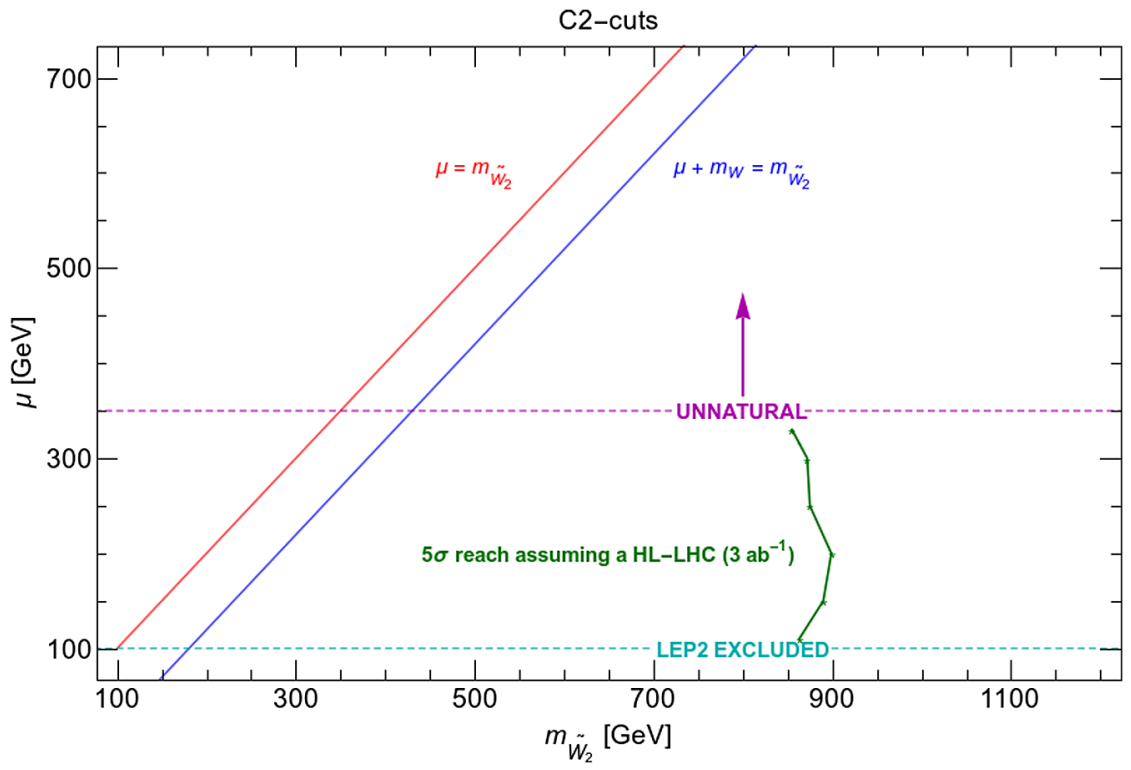

FIG. 7. Discovery reach in the SSdB channel at the HL-LHC in the $m_{\tilde{W}_{2}}$ vs $\mu$ plane. 
unified gaugino mass $m_{1 / 2}$ of $\sim 1010(1280) \mathrm{GeV}$. In terms of the comparable reach in terms of $m_{\tilde{g}}$, these correspond to $m_{\tilde{g}} \sim 2430$ (3000) GeV. These values may be compared to the $5 \sigma 3 \mathrm{ab}^{-1}$ HL-LHC for direct gluino pair production of $m_{\tilde{g}} \sim 2800 \mathrm{GeV}$ obtained in Ref. [24]. Although we do not show it on the figure, we mention that with the hard C2 cuts, the discovery reach of the LHC extends to $500 \mathrm{GeV}$ $(720 \mathrm{GeV})$ for an integrated luminosity of $300 \mathrm{fb}^{-1}$ $\left(1 \mathrm{ab}^{-1}\right)$, while the corresponding 95\% CL exclusion extends to $780 \mathrm{GeV}(980 \mathrm{GeV})$. It is worth keeping in mind that especially for the $300 \mathrm{fb}^{-1}$ case, somewhat softer analysis cuts $[26,29]$ may be better suited for optimizing the LHC reach.

The key mass relation for the SSdB signature is that $|\mu| \ll M_{2}$. It is therefore interesting to explore our discovery reach beyond our benchmark assumption of $|\mu|=150 \mathrm{GeV}$. In Fig. 7 , we denote the $\left(3 \mathrm{ab}^{-1}\right) \mathrm{HL}-$ LHC $(5 \sigma)$ discovery reach in the $\mu-M_{2}$ plane by the green solid line in the vicinity of $m_{\tilde{W}_{2}} \simeq 850-900 \mathrm{GeV}$. As expected the reach is only weakly sensitive to the higgsino mass. The red diagonal line in Fig. 7 shows where $\mu=m_{\tilde{W}_{2}}$. Above this line the $\mathrm{SSdB}$ signature arises from higgsino pair production and subsequent decays to winos; but it would have a much smaller rate because (1) the higgsino cross section is smaller than the wino cross section, and (2) dilution of the signal from higgsino decays to binos (if these are accessible). Below the blue diagonal line in Fig. 7 denotes the region where $\tilde{W}_{2} \rightarrow \tilde{Z}_{1,2}+W$ or $\tilde{Z}_{4} \rightarrow \tilde{W}_{1}+W$ decays can occur, leading the SSdB final state, with on-shell $W$ s. Close to this line and for not-toolarge $m_{\tilde{W}_{2}}$, though, the same sign dilepton events would not necessarily be clean as the large wino-higgsino mixings would lead to sizeable mass gaps and concomitant harder debris from the decay of the lighter inos. As $\mu$ increases, the model becomes increasingly unnatural, with a value $\mu>$ 350 (indicated by a magenta dashed line) corresponding to electroweak fine-tuning measure $\Delta_{\mathrm{EW}}>30$. The natural SUSY region is the region below this horizontal line.

\section{SSdB SUSY EVENT CHARACTERISTICS}

We have already illustrated the $E_{T}$ and lepton transverse momentum distributions after all cuts in Fig. 4 and Fig. 5, respectively. We saw that while the $E_{T}$ distribution from signal emerges from the background for $\mathscr{E}_{T}>250 \mathrm{GeV}$, this distribution is typically backed up against the cut. Although the distribution may harden somewhat with increasing wino mass, we saw that the observability of the signal becomes rate limited by the time we reach $m_{\tilde{W}_{2}}=860 \mathrm{GeV}$, so wino events would typically have $E_{T} \sim 250-500 \mathrm{GeV}$. The lepton $p_{T}$ distributions peak at $200-250 \mathrm{GeV}$ for the hard lepton and $50-100 \mathrm{GeV}$ for the second lepton, independent of the wino mass. This should not be very surprising because the leptons are produced at the end of a cascade decay chain, so the $p_{T \ell}$ distributions are only altered by the changes in the boost of the daughter $W$ bosons which share the parent wino energy with the (nearly invisible) higgsinos.

To further characterize the nature of the SSdB events from SUSY, and to see if we can gain some sensitivity to the wino mass from the kinematic properties of these events, we have examined several kinematic variables: $A_{\text {eff }}$, $m_{T}^{\min }$ (which entered the $\mathbf{C 1}$ cuts), its sibling $m_{T}^{\max }, m_{T 2}$, $m_{C T}$ and $m_{\ell \ell}$, where

$$
A_{\text {eff }}=\mathscr{E}_{T}+\sum_{i}^{n(j)} p_{T}\left(j_{i}\right)+p_{T}\left(\ell_{1}\right)+p_{T}\left(\ell_{2}\right),
$$

and $m_{C T}$ is the cluster transverse mass given by

$$
m_{C T}^{2}=m_{C T}^{2}=\left(\not_{T}+\sqrt{\vec{p}_{T \ell \ell}^{2}+m_{\ell \ell}^{2}}\right)^{2}-\left(\vec{E}_{T}+\vec{p}_{T \ell \ell}\right)^{2} .
$$

In Fig. 8, we show the normalized distributions of $m_{T}^{\min }$ (because it enters our analysis cuts) together with those of $A_{\text {eff }}, m_{C T}$, and $m_{T}^{\max }$, the larger of the transverse masses of the lepton and $E_{T}$. These are the distributions whose shapes show the most sensitivity to the wino mass for the three benchmark SUSY cases introduced above. We see that even for these three cases with a fairly wide separation of wino masses, the shapes of the distributions are qualitatively quite similar, with perhaps the $m_{T}^{\max }$ distribution showing the greatest sensitivity to the parent wino mass. As we noted in the discussion of Fig. 5, the wino mass has a relatively small effect on the kinematics of signal events, affecting only the boost of the $W$ bosons. While these (quite correlated) distributions show some differences, especially in the tails of the distributions which correspond to relatively low numbers of signal events, we will see below that because the signal rate can be predicted with good precision, the event rate for the SSdB signal offers a much better handle on the wino mass. We stress, though, that the kinematic properties of these events are nonetheless useful for validating the signal origin, and could potentially serve as ingredients in an artificial neural network stew.

The charge asymmetry

$$
A=\frac{n(++)-n(--)}{n(++)+n(--)}
$$

of clean same sign dilepton events (which, of course, includes both signal and background events) provides yet another handle for validating the wino origin of any signal. We show a fit to the expected $A$ values (our simulated sample had considerable statistical fluctuations) for signalplus-background events versus $m_{\tilde{W}_{2}}$ in Fig. 9, together with the expected background value. The charge asymmetry arises because there are more up-type than down-type valence quarks in a proton. The importance of valence quark collisions for wino pair production processes 

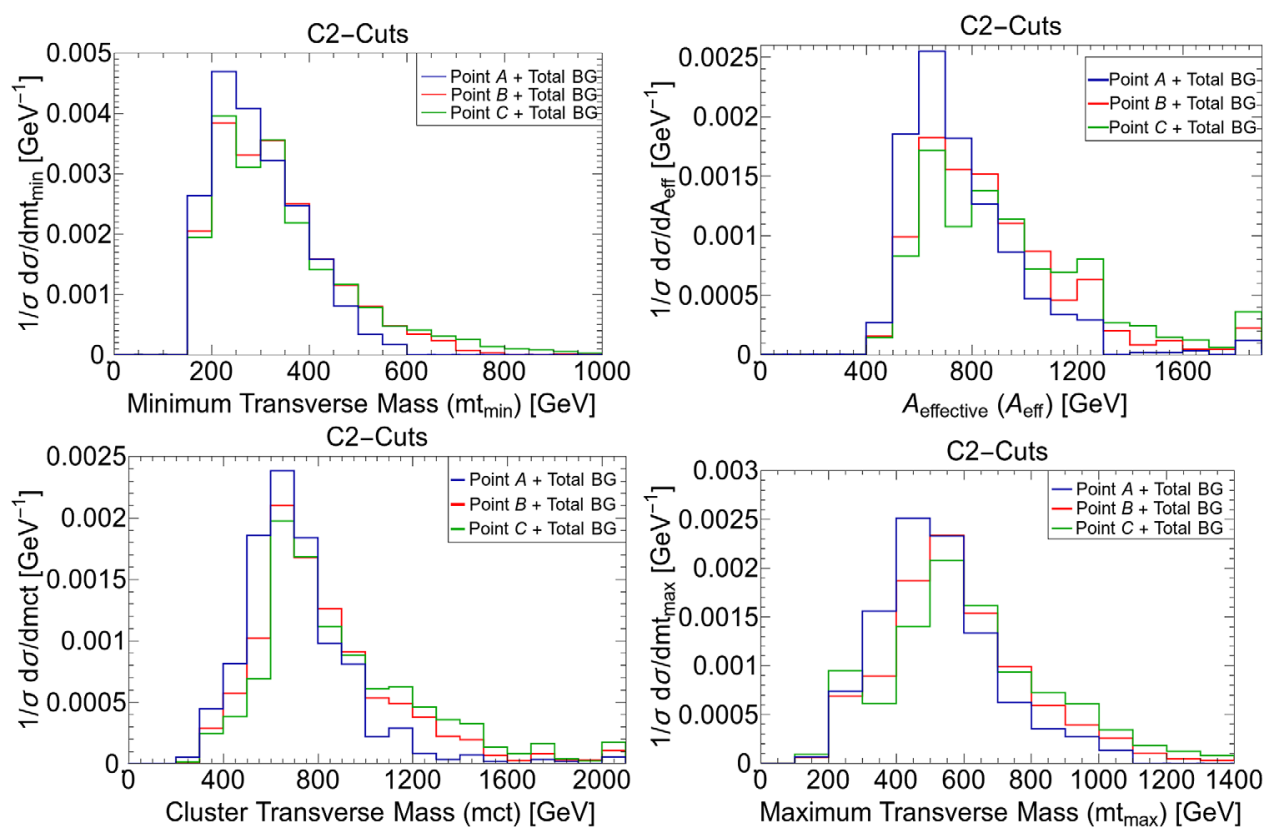

FIG. 8. Distributions of $m_{T}^{\min }$ (top left), $A_{\text {eff }}$ (top right), $m_{C T}$ (bottom left) and $m_{T}^{\max }$ (bottom right) from the SUSY SSdB signal plus SM backgrounds after $\mathbf{C} 2$ cuts for the three benchmark cases Point A, Point B, and Point C introduced earlier in the text. We have normalized these distributions to all have the same area.

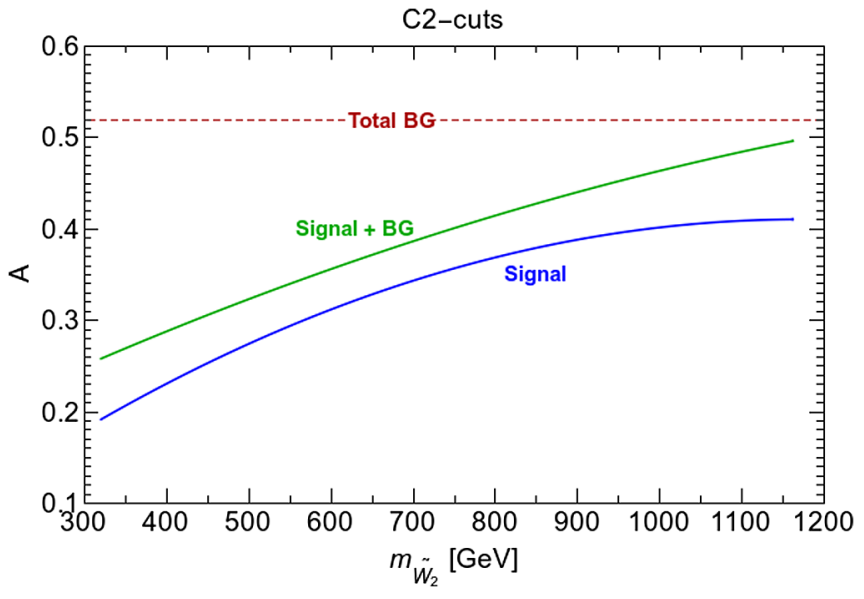

FIG. 9. Same-sign dilepton charge asymmetry from signalplus-background vs $m_{\tilde{W}_{2}}$ from SUSY same-sign diboson production after C2 cuts versus $m_{\tilde{W}_{2}}$ at LHC with $\sqrt{s}=14 \mathrm{TeV}$. The statistical error with which the charge asymmetry can be determined is $\sim \pm 0.1$ is $m_{\tilde{W}_{2}} \lesssim 800 \mathrm{GeV}$.

increases with wino mass, so we expect the asymmetry to also increase with $m_{\tilde{W}_{2}}$. This is indeed borne out in the figure where we see that the expected asymmetry ranges from 0.2 for $m_{\tilde{W}_{2}}$ as low as $\sim 300 \mathrm{GeV}$ to 0.4 for $m_{\tilde{W}_{2}} \sim 1000 \mathrm{GeV}{ }^{15}$ Unfortunately, the measured charge asymmetry does not provide as good of a wino mass

\footnotetext{
${ }^{15}$ The asymmetry of the background is even larger because the $W^{ \pm} W^{ \pm} j j$ component of the background, though subdominant, has contributions from collisions of two valence quarks.
}

determination as one might naively suppose from looking at the figure. The reason is that because of the relatively low total event rate, even with $3 \mathrm{ab}^{-1}$, the statistical error on its measurement is $\sim \pm 0.1$ for $m_{\tilde{W}_{2}}<800 \mathrm{GeV}$, which corresponds to a wino mass uncertainty of $\sim 300 \mathrm{GeV}$. We nevertheless stress that a determination of the charge asymmetry provides a consistency check of wino origin of the SSdB signal if $m_{\tilde{W}_{2}}$ can be extracted from the total event rate. An examination of this extraction is the subject of the next section.

\section{MEASUREMENT OF THE WINO MASS IN THE SSdB CHANNEL}

We saw that while experiments at the HL-LHC would be able to discover winos with masses up to $860 \mathrm{GeV}$ and to exclude these out to $1100 \mathrm{GeV}$ if no excess is seen, the determination of its mass from the kinematic properties of the signal event proved rather difficult. We traced this to the fact that the leptons were produced only at the end of a cascade so that the sensitivity to the mass of the parent winos is correspondingly reduced.

In principle, it should also be possible to determine the wino mass from the rate with which the signal events are produced. This is particularly true in this case because the cross section for wino production can be rather precisely computed for the case of natural SUSY (for which the heavier inos are expected to be nearly pure gauginos) and depends on just the wino mass. We also saw in Sec. II B that, at least for $m_{\tilde{W}_{2}}>500 \mathrm{GeV}$, the natural SUSY branching fraction for wino decays to $W$ is $0.25 \pm 0.02$ 
with conservative error bars. ${ }^{16}$ The determination of the SSdB signal rate after C2 cuts shown in Fig. 6 thus provides a plausible mass measurement strategy, because, to a good approximation, the observed number of events depends only on the wino mass.

For example, for our assumed benchmark point, Point $\mathrm{B}$, and using $\mathbf{C 2}$ cuts, with $3 \mathrm{ab}^{-1}$ we expect a total of $63 \pm 8$ events (see Table II), where the error bar is purely statistical. Since we would estimate the signal cross section by taking the observed number of events and subtracting the expected number of background events, this \pm 8 events corresponds to $\mathrm{a} \approx 16 \%$ measurement of the cross section, which, as one can find by examining the cross section after C2 cuts (as in Fig. 6) corresponds to a measurement of $m_{\tilde{W}_{2}} \sim 690 \pm 35 \mathrm{GeV}$, which represents a better than $5 \%$ measurement of the wino mass.

This precision is possible when we consider statistical errors alone. There is also a systematic error arising from the theory uncertainty on the cross section, uncertainties on the wino decay branching ratios, uncertainties on the efficiencies for events passing cuts, uncertainties on the reconstruction efficiencies, etc. Since the current uncertainty ( $\sim 10 \%$ in the production cross section) mostly arises from the uncertainties in the parton distributions which will undoubtedly be well measured by the time this analysis is done, and the lepton detection efficiencies will also be well understood, we expect the main systematic will arise from the squared wino branching fraction, which as we have already noted is $\lesssim 16 \%$. Conservatively taking the total systematic to be $\sim 20 \%$, then our error on the wino mass for Point B increases to $\approx 50 \mathrm{GeV}$. Even if the total systematic error on the cross section is $30 \%$, then the combined statistical and systematic error on the mass is $\approx 70 \mathrm{GeV}$, which is about a $10 \%$ measurement of the wino mass. If our background is underestimated by a factor of two, our measurement of the wino mass will be biased by $\approx 70 \mathrm{GeV}$ toward lower values; if it is overestimated by a factor of two, then our measurement will be biased by $\approx 35 \mathrm{GeV}$ toward higher values.

We can still make a good mass measurement for large values of the wino mass; for instance, the purely statistical error on the mass measurement is still only $\approx 10 \%$ for a $1 \mathrm{TeV}$ wino (although there is no $5 \sigma$ signal). However for these larger mass values with their correspondingly smaller signal cross sections, very precise determinations of the background cross section become increasingly important. Presumably, these will be experimentally determined by an extrapolation into the signal region by the time the

\footnotetext{
${ }^{16}$ As we have already noted, the observation of a signal in the clean, same sign dilepton channel already points to light higgsinos and much heavier EW gauginos. Additional circumstantial evidence for light higgsinos could, for instance, come from the observation of monojet plus soft dilepton events, which must be present at observable rates if $m_{\tilde{Z}_{2}}-m_{\tilde{Z}_{1}} \gtrsim 10 \mathrm{GeV}$ and higgsinos are not much heavier than $220-240 \mathrm{GeV}$.
}

HL-LHC accumulates $3 \mathrm{ab}^{-1}$ of data. Our point is that better than $10 \%$ determination of the wino mass will be possible if the SSdB signal from natural SUSY is detected at the HL-LHC.

\section{CONCLUSIONS}

In this paper we have revisited and explored aspects of the SSdB signature, which is a powerful channel for discovering natural SUSY models with $|\mu| \ll M_{2}$, especially if $M_{3}$ is larger than in unified models. This signature arises from wino pair production, $p p \rightarrow \tilde{W}_{2} \tilde{Z}_{4}$, followed by wino decays to $W$ bosons plus quasivisible higgsinos. Thus, the signal consists of $\ell^{ \pm} \ell^{ \pm \pm}+E_{T}$ events which are distinct from same-sign dilepton events from gluino/squark production in that they are relatively free of hard jet activity. We emphasize that the SSdB search channel offers a probe of natural SUSY - indeed of all SUSY models with light higgsinos - that is independent of any signals from gluino pair or top-squark pair production. The SSdB channel is especially useful because (i) SM backgrounds for such a signature are tiny and (ii) this type of signature is not expected in many previously studied "unnatural" SUSY models, such as mSUGRA/CMSSM, where the opposite mass hierarchy, $M_{2}<|\mu|$, and $M_{1}<M_{2}$ is expected.

We have evaluated several new background contributions to the SSdB signature including $W W j j$ production, $4 t$ production, and $3 W$ production. We find these new background reactions can be suppressed beyond the previously examined $\mathbf{C} \mathbf{1}$ cuts by an additional jet veto $n$ (jets) $\leq 1$ and a hardened $\not_{T}$ cut at a modest cost to the signal. The surviving signal rate should be observable at HL-LHC with $3 \mathrm{ab}^{-1}$ of integrated luminosity over a large range of wino mases. After our $\mathbf{C 2}$ analysis cuts, the HL-LHC $5 \sigma$ reach (95\% CL exclusion) extends out to $m_{\tilde{W}_{2}}=860 \mathrm{GeV}$ $(1080 \mathrm{GeV})$. We show that a determination of the clean same sign dilepton event rate allows a better than $10 \%$ measurement of the wino mass over the entire range of masses for which experiments at the HL-LHC will be able to discover a wino in this channel. A measurement of the like-sign dilepton lepton charge asymmetry will test the consistency of the wino origin of the signal. If gluinos are also discovered at the HL-LHC, experiments will be able to probe whether or not gaugino masses arise from a common mass at $Q \simeq M_{\mathrm{GUT}}$ at the $10 \%$ level [24]. We encourage continued experimental scrutiny of the clean same sign dilepton $+E_{T}$ channel as the integrated luminosity at the LHC goes beyond $\sim 100 \mathrm{fb}^{-1}$.

\section{ACKNOWLEDGMENTS}

This work was supported in part by the US Department of Energy, Office of High Energy Physics and was performed in part at the Aspen Center for Physics, which is supported by National Science Foundation Grant No. PHY-1607611. 
[1] The ATLAS Collaboration, Report No. ATLAS-CONF2017-022, http://cdsweb.cern.ch/record/2258145; T. Sakuma (CMS Collaboration), Squark/gluino searches in hadronic channels with CMS, Proc. Sci., LHCP2016 (2017) 145 [arXiv:1609.07445].

[2] The ATLAS Collaboration, Report No. ATLAS-CONF2017-037, https://cds.cern.ch/record/2266170; A. M. Sirunyan et al. (CMS Collaboration), Search for top squark pair production in pp collisions at $\sqrt{s}=13 \mathrm{TeV}$ using single lepton events, J. High Energy Phys. 10 (2017) 019.

[3] N. Craig, The state of supersymmetry after Run I of the LHC, arXiv:1309.0528.

[4] R. Barbieri and G. F. Giudice, Upper bounds on supersymmetric particle masses, Nucl. Phys. B306, 63 (1988).

[5] R. Kitano and Y. Nomura, Supersymmetry, naturalness, and signatures at the CERN LHC, Phys. Rev. D 73, 095004 (2006); M. Papucci, J. T. Ruderman, and A. Weiler, Natural SUSY endures, J. High Energy Phys. 09 (2012) 035.

[6] H. Baer, V. Barger, and D. Mickelson, How conventional measures overestimate electroweak fine-tuning in supersymmetric theory, Phys. Rev. D 88, 095013 (2013).

[7] A. Mustafayev and X. Tata, Supersymmetry, naturalness and light higgsinos, Indian J. Phys. 88, 991 (2014).

[8] H. Baer, V. Barger, D. Mickelson, and M. PadeffkeKirkland, SUSY models under siege: LHC constraints and electroweak fine-tuning, Phys. Rev. D 89, 115019 (2014).

[9] H. Baer, V. Barger, P. Huang, D. Mickelson, A. Mustafayev, and X. Tata, Radiative natural supersymmetry: Reconciling electroweak fine-tuning and the Higgs boson mass, Phys. Rev. D 87, 115028 (2013).

[10] H. Baer, V. Barger, P. Huang, A. Mustafayev, and X. Tata, Radiative Natural Supersymmetry with a $125 \mathrm{GeV}$ Higgs Boson, Phys. Rev. Lett. 109, 161802 (2012).

[11] For a recent overview of upper bounds on stop and gluino masses in a variety of models from the requirement $\Delta_{\text {EW }}<30$, see Sec. V of H. Baer, V. Barger, J. Gainer, H. Serce, and X. Tata, Reach of the high-energy LHC for gluinos and top squarks in SUSY models with light Higgsinos, Phys. Rev. D 96, 115008 (2017).

[12] C. Brust, A. Katz, S. Lawrence, and R. Sundrum, SUSY, the third generation and the LHC, J. High Energy Phys. 03 (2012) 103.

[13] L. Girardello and M. T. Grisaru, On the MSSM Higgsino mass and fine tuning, Nucl. Phys. B194, 65 (1982); and recently re-emphhasized by G. G. Ross, K. SchmidtHoberg, and F. Staub, Phys. Lett. B 759, 110 (2016).

[14] T. Cohen, J. Kearney, and M. Luty, Natural supersymmetry without light Higgsinos, Phys. Rev. D 91, 075004 (2015); A. Nelson and T. Roy, Generalized Supersoft Supersymmetry Breaking and a Solution to the $\mu$ Problem, Phys. Rev. Lett. 114, 201802 (2015); S. P. Martin, Nonstandard supersymmetry breaking and Dirac gaugino masses without supersoftness, Phys. Rev. D 92, 035004 (2015).

[15] J. R. Ellis, K. Enqvist, D. V. Nanopoulos, and F. Zwirner, Observables in low-energy superstring models, Mod. Phys. Lett. A 01, 57 (1986).

[16] H. Baer, V. Barger, P. Huang, D. Mickelson, M. PadeffkeKirkland, and X. Tata, Natural SUSY with a bino- or winolike LSP, Phys. Rev. D 91, 075005 (2015).
[17] CMS Collaboration, Report No. CMS-PAS-SUS-17-004, https://cds.cern.ch/record/2273907; ATLAS Collaboration, Report No. ATLAS-CONF-2017-039, https://cds.cern.ch/ record/2267406.

[18] R. D. Peccei and H. R. Quinn, CP Conservation in the Presence of Pseudoparticles, Phys. Rev. Lett. 38, 1440 (1977); Constraints imposed by $C P$ conservation in the presence of pseudoparticles, Phys. Rev. D 16, 1791 (1977); S. Weinberg, A New Light Boson?, Phys. Rev. Lett. 40, 223 (1978); F. Wilczek, Problem of Strong $P$ and $T$ Invariance in the Presence of Instantons, Phys. Rev. Lett. 40, 279 (1978).

[19] K. J. Bae, H. Baer, and E. J. Chun, Mainly axion cold dark matter from natural supersymmetry, Phys. Rev. D 89, 031701 (2014); Mixed axion/neutralino dark matter in the SUSY DFSZ axion model, J. Cosmol. Astropart. Phys. 12 (2013) 028.

[20] H. Baer, A. Mustafayev, and X. Tata, Monojets and monophotons from light higgsino pair production at LHC14, Phys. Rev. D 89, 055007 (2014); See also, C. Han, A. Kobakhidze, N. Liu, A. Saavedra, L. Wu, and J. M. Yang, Probing light higgsinos in natural SUSY from monojet signals at the LHC, J. High Energy Phys. 02 (2014) 049; P. Schwaller and J. Zurita, Compressed electroweakino spectra at the LHC, J. High Energy Phys. 03 (2014) 060.

[21] Z. Han, G. D. Kribs, A. Martin, and A. Menon, Hunting quasidegenerate Higgsinos, Phys. Rev. D 89, 075007 (2014); H. Baer, A. Mustafayev, and X. Tata, Monojet plus soft dilepton signal from light higgsino pair production at LHC14, Phys. Rev. D 90, 115007 (2014); C. Han, D. Kim, S. Munir, and M. Park, Accessing the core of naturalness, nearly degenerate higgsinos, at the LHC, J. High Energy Phys. 04 (2015) 132.

[22] H. Baer, V. Barger, D. Mickelson, A. Mustafayev, and X. Tata, Physics at a higgsino factory, J. High Energy Phys. 06 (2014) 172.

[23] H. Baer, M. Berggren, K. Fujii, S. L. Lehtinen, J. List, T. Tanabe, and J. Yan, Naturalness and light higgsinos: A powerful reason to build the ILC, Proc. Sci., ICHEP2016 (2016) 156 [arXiv:1611.02846].

[24] H. Baer, V. Barger, J. S. Gainer, P. Huang, M. Savoy, D. Sengupta, and $\mathrm{X}$. Tata, Gluino reach and mass extraction at the LHC in radiatively-driven natural SUSY, Eur. Phys. J. C 77, 499 (2017).

[25] H. Baer, V. Barger, N. Nagata, and M. Savoy, Phenomenological profile of top squarks from natural supersymmetry at the LHC, Phys. Rev. D 95, 055012 (2017).

[26] H. Baer, V. Barger, P. Huang, D. Mickelson, A. Mustafayev, W. Sreethawong, and X. Tata, Radiatively-driven natural supersymmetry at the LHC, J. High Energy Phys. 12 (2013) 013; Erratum, J. High Energy Phys.06 (2015) 053.

[27] B. Altunkaynak, H. Baer, V. Barger, and P. Huang, Distinguishing LSP archetypes via gluino pair production at LHC13, Phys. Rev. D 92, 035015 (2015).

[28] CMS Collaboration, LHC BSM searches, see talk by J. Hirschauer, Pheno2017 meeting, Pittsburgh, PA, May, 2017, https://indico.cern.ch/event/610112/contributions/2570016/.

[29] H. Baer, V. Barger, P. Huang, D. Mickelson, A. Mustafayev, W. Sreethawong, and X. Tata, Same-Sign Diboson Signature from Supersymmetry Models with Light Higgsinos at the LHC, Phys. Rev. Lett. 110, 151801 (2013). 
[30] R. M. Barnett, J. F. Gunion, and H. E. Haber, Reports No. UCD-88-30, No. LBL-26204, and No. SCIPP-88-36; H. Baer, X. Tata, and J. Woodside, Gluino-cascade-decay signatures at the Fermilab Tevatron collider, Phys. Rev. D 41, 906 (1990); Multilepton signals from supersymmetry at hadron supercolliders, Phys. Rev. D 45, 142 (1992); R. M. Barnett, J. F. Gunion, and H. E. Haber, Discovering supersymmetry with like-sign dileptons, Phys. Lett. B 315, 349 (1993).

[31] D. Matalliotakis and H. P. Nilles, Implications of nonuniversality of soft terms in supersymmetric grand unified theories, Nucl. Phys. B435, 115 (1995); M. Olechowski and S. Pokorski, Electroweak symmetry breaking with nonuniversal scalar soft terms and large $\tan \beta$ solutions, Phys. Lett. B 344, 201 (1995); P. Nath and R. L. Arnowitt, Nonuniversal soft supersymmetry breaking and dark matter, Phys. Rev. D 56, 2820 (1997); J. Ellis, K. Olive, and Y. Santoso, The MSSM parameter space with non-universal Higgs masses, Phys. Lett. B 539, 107 (2002); J. Ellis, T. Falk, K. Olive, and Y. Santoso, Exploration of the MSSM with non-universal Higgs masses, Nucl. Phys. B652, 259 (2003); H. Baer, A. Mustafayev, S. Profumo, A. Belyaev, and X. Tata, Direct, indirect and collider detection of neutralino dark matter in SUSY models with non-universal Higgs masses, J. High Energy Phys. 07 (2005) 065.

[32] U. Chattopadhyay, A. Datta, A. Datta, A. Datta, and D. P. Roy, LHC signature of the minimal SUGRA model with a large soft scalar mass, Phys. Lett. B 493, 127 (2000); P. Mercadante, J. K. Mizukoshi, and X. Tata, Using $b$-tagging to enhance the supersymmetry reach of the CERN Large Hadron Collider, Phys. Rev. D 72, 035009 (2005); R. H. K. Kadala, P. Mercadante, J. K. Mizukoshi, and X. Tata, Heavy-flavor tagging and the supersymmetry reach of the CERN Large Hadron Collider, Eur. Phys. J. C 56, 511 (2008).

[33] H. Baer, V. Barger, M. Savoy, and X. Tata, Multichannel assault on natural supersymmetry at the high luminosity LHC, Phys. Rev. D 94, 035025 (2016).

[34] H. Baer, V. Barger, H. Serce, and X. Tata, Natural generalized mirage mediation, Phys. Rev. D 94, 115017 (2016).

[35] H. Baer, V. Barger, M. Savoy, H. Serce, and X. Tata, Superparticle phenomenology from the natural minilandscape, J. High Energy Phys. 06 (2017) 101.

[36] J. Alwall, M. Herquet, F. Maltoni, O. Mattelaer, and T. Stelzer, MadGraph 5: Going beyond, J. High Energy Phys. 06 (2011) 128; J. Alwall, R. Frederix, S. Frixone, V Herschi, F. Maltoni, O. Mattelaer, H.-S. Shao, T. Stelzer, P. Torrielli, and M. Zaro, The automated computation of tree-level and next-to-leading order differential cross sections, and their matching to parton shower simulations, J. High Energy Phys. 07 (2014) 079.

[37] T. Sjöstrand, S. Ask, J. R. Christiansen, R. Corke, N. Desai, P. Ilten, S. Mrenna, S. Prestel, C. O. Rasmussen, and P. Z. Skands, An introduction to PYTHIA 8.2, Comput. Phys. Commun. 191, 159 (2015).

[38] J. de Favereau, C. Delaere, P. Demin, A. Giammanco, V. Lemaître, A. Mertens, and M. Selvaggi (DELPHES 3
Collaboration), DELPHES 3, A modular framework for fast simulation of a generic collider experiment, J. High Energy Phys. 02 (2014) 057.

[39] W. Beenakker, R. Hopker, and M. Spira, PROSPINO: A program for the production of supersymmetric particles in next-to-leading order QCD, arXiv:hep-ph/9611232.

[40] H. Baer and X. Tata, Weak Scale Supersymmetry (Cambridge University Press, Cambridge, England, 2006).

[41] F. E. Paige, S. D. Protopopescu, H. Baer, and X. Tata, ISAJET 7.69: A Monte Carlo event generator for pp, anti-p p, and e+e- reactions, arXiv:hep-ph/0312045.

[42] M. Czakon, P. Fiedler, and A. Mitov, Total Top-Quark PairProduction Cross Section at Hadron Colliders Through $O\left(\alpha_{s}^{4}\right)$, Phys. Rev. Lett. 110, 252004 (2013).

[43] G. Bevilacqua and M. Worek, Constraining BSM physics at the LHC: Four top final states with NLO accuracy in perturbative QCD, J. High Energy Phys. 07 (2012) 111.

[44] J. M. Campbell, R. K. Ellis, and C. Williams, Vector boson pair production at the LHC, J. High Energy Phys. 07 (2011) 018.

[45] J. M. Campbell and R. K. Ellis, $t \bar{t} W^{ \pm}$production and decay at NLO, J. High Energy Phys. 07 (2012) 052.

[46] A. Kardos, Z. Trócsányi, and C. Papadopoulos, Top quark pair production in association with a Z-boson at nextto-leading-order accuracy, Phys. Rev. D 85, 054015 (2012).

[47] T. Melia, K. Melnikov, R. Röntsch, and G. Zanderighi, Next-to-leading order QCD predictions for $W^{+} W^{+} j j$ production at the LHC, J. High Energy Phys. 12 (2010) 053.

[48] Y. B. Shen, R. Y. Zhang, W. G. Ma, X. Z. Li, and L. Guo, NLO QCD and electroweak corrections to WWW production at the LHC, Phys. Rev. D 95, 073005 (2017).

[49] M. Cacciari, G. P. Salam, and G. Soyez, The anti- $k_{t}$ jet clustering algorithm, J. High Energy Phys. 04 (2008) 063.

[50] M. Cacciari, G. P. Salam, and G. Soyez, FastJet user manual, Eur. Phys. J. C 72, 1896 (2012).

[51] CMS Collaboration, Report No. CMS-PAS-BTV-15-001, https://cds.cern.ch/record/2138504.

[52] M. Cacciari and G. P. Salam, Pileup subtraction using jet areas, Phys. Lett. B 659, 119 (2008).

[53] S. Corréard, V. Kostyukhin, J. Lévêque, A. Rozanov, and J. B. De Vivie de Régie, ATLAS Report No. ATLAS-PHYS2004-006, https://cds.cern.ch/record/686346; V. Kostyukhin, ATLAS Report No. ATLAS-PHYS-2003-033, http://cds.cern .ch/record/685550.

[54] V. D. Barger, T. Han, and R. J. N. Phillips, Improved transverse-mass variable for detecting Higgs-boson decays into Z pairs, Phys. Rev. D 36, 295 (1987).

[55] C. G. Lester and D. J. Summers, Measuring masses of semi-invisibly decaying particle pairs produced at hadron colliders, Phys. Lett. B 463, 99 (1999); A. Barr, C. Lester, and P. Stephens, A variable for measuring masses at hadron colliders when missing energy is expected; $m_{T 2}$ : the truth behind the glamour, J. Phys. G 29, 2343 (2003). 